OPEN ACCESS

Edited by:

Aneta Kielar.

University of Arizona, United States

Reviewed by:

Julie A. Onton,

University of California, San Diego, United States

Görsev Yener,

Dokuz Eylul University, Turkey Regina Maria Baratho, Pontifical Catholic University of São Paulo, Brazil

*Correspondence: Jaeuk U. Kim jaeukkim@kiom.re.kr

tThese authors have contributed equally to this work

Received: 28 January 2021 Accepted: 19 March 2021

Published: 13 April 2021

Citation:

Doan DNT, Ku B, Choi J, Oh M, Kim K, Cha W and Kim JU (2021) Predicting Dementia With Prefrontal Electrencephalography and Event-Related Potential. Front. Aging Neurosci. 13:659817. doi: 10.3389/fnagi.2021.659817

\section{Predicting Dementia With Prefrontal Electroencephalography and Event-Related Potential}

\author{
Dieu Ni Thi Doan ${ }^{1,2+}$, Boncho $\mathrm{Ku}^{1 \dagger}$, Jungmi Choi ${ }^{3}$, Miae $\mathrm{Oh}^{4}$, Kahye Kim ${ }^{1}$, Wonseok Cha ${ }^{3}$ \\ and Jaeuk U. Kim ${ }^{1,2 *}$
}

${ }^{1}$ Korea Institute of Oriental Medicine, Daejeon, South Korea, ${ }^{2}$ Korean Convergence Medicine, University of Science and Technology, Daejeon, South Korea, ${ }^{3}$ Human Anti-Aging Standards Research Institute, Uiryeong-gun, South Korea, ${ }^{4}$ Korea Institute for Health and Social Affairs, Sejong, South Korea

Objective: To examine whether prefrontal electroencephalography (EEG) can be used for screening dementia.

Methods: We estimated the global cognitive decline using the results of Mini-Mental Status Examination (MMSE), measurements of brain activity from resting-state EEG, responses elicited by auditory stimulation [sensory event-related potential (ERP)], and selective attention tasks (selective-attention ERP) from 122 elderly participants (dementia, 35; control, 87). We investigated that the association between MMSE and each EEG/ERP variable by using Pearson's correlation coefficient and performing univariate linear regression analysis. Kernel density estimation was used to examine the distribution of each EEG/ERP variable in the dementia and non-dementia groups. Both Univariate and multiple logistic regression analyses with the estimated odds ratios were conducted to assess the associations between the EEG/ERP variables and dementia prevalence. To develop the predictive models, five-fold cross-validation was applied to multiple classification algorithms.

Results: Most prefrontal EEG/ERP variables, previously known to be associated with cognitive decline, show correlations with the MMSE score (strongest correlation has $|r|=0.68)$. Although variables such as the frontal asymmetry of the resting-state EEG are not well correlated with the MMSE score, they indicate risk factors for dementia. The selective-attention ERP and resting-state EEG variables outperform the MMSE scores in dementia prediction (areas under the receiver operating characteristic curve of 0.891, 0.824 , and 0.803 , respectively). In addition, combining EEG/ERP variables and MMSE scores improves the model predictive performance, whereas adding demographic risk factors do not improve the prediction accuracy.

Conclusion: Prefrontal EEG markers outperform MMSE scores in predicting dementia, and additional prediction accuracy is expected when combining them with MMSE scores.

Significance: Prefrontal EEG is effective for screening dementia when used independently or in combination with MMSE.

Keywords: dementia, Alzheimer's disease, electroencephalography, electrophysiology, event-related potential, Mini-Mental Status Examination 


\section{INTRODUCTION}

Dementia is a clinical syndrome that comprises a group of neurodegenerative disorders related to cognitive decline that influence memory, language presentation, social abilities, and executive functions, et cetera (McKhann et al., 2011; DSM-5). With the progression of cognitive decline, dementia patients gradually experience memory deficits, communication disorders, and difficulty performing activities of daily living and eventually become fully dependent on caregivers (Chertkow et al., 2013). Alzheimer's disease $(\mathrm{AD})$ is the most common cause of dementia, representing $60 \%-70 \%$ of cases. Other common causes of dementia include cerebrovascular disease, Lewy Bodies disease, and frontotemporal dementia (World Health Organization, 2020).

Aging is the major risk factor for dementia, which has a prevalence of approximately $97 \%$ in population aged 65 years and above (Alzheimer's Association Report, 2020). The increasing world population and life expectancy have led to a rapid increase in the number of dementia patients, which is estimated to reach 82 million people worldwide by 2030 (World Health Organization, 2020). A substantial burden on social care and degradation of quality of life may follow. Furthermore, the deaths attributed to $\mathrm{AD}$ have positioned this condition as the fifth leading cause of death globally, causing 122,019 deaths in 2018 alone (Alzheimer's Association Report, 2020).

Although no known treatment is highly effective for any type of dementia, combined therapeutic tools which are available to mitigate the after effects of cognitive impairment, especially during the early stages of these diseases (Robinson et al., 2015; Tisher and Salardini, 2019). Moreover, the effectiveness of early therapeutic interventions can be increased to achieve disease modification when neuronal degeneration has not yet begun (Sperling et al., 2011; Tisher and Salardini, 2019). As the disease progresses, neurons accumulate abnormal proteins, such as beta-amyloid and tau proteins, and exhibit mitochondrial dysfunction and calcium homeostasis dysregulation (Niedowicz et al., 2011; Kocahan and Doan, 2017; Farooqui, 2019). In the later stages, the brain of the patient presents neuroinflammation and irreversible synaptic loss, leading to neuronal death and brain tissue damage (Niedowicz et al., 2011; Kocahan and Doan, 2017; Farooqui, 2019).

Early detection of neuronal damage in the brain that enables both timely therapeutic intervention to manage the symptoms and adequate preparation of patients and caregivers. Early prediction of dementia is possible when the underlying disease is defined with tangible biomarkers. Recently, the national institute on aging and the Alzheimer's association proposed an $\mathrm{AD}$ research framework using diagnostic biomarkers that are standardized in terms of beta-amyloid deposition, pathologic tau, and neurodegeneration, representing a shift from syndrome to biological constructions (Sperling et al., 2011; Jack et al., 2018). Beta-amyloid plaques and neurofibrillary tau tangles uniquely characterize $\mathrm{AD}$ among various neurodegenerative disorders that may progress to dementia (McKhann et al., 2011; Jack et al., 2018). Although these biomarker profiles are stated as core neuropathologic changes for defining $\mathrm{AD}$ and related terms in the research framework, they remain incomplete and inadequate for clinical practice (Jack et al., 2018). Furthermore, they are clinically accessible only at advanced hospitals and are frequently costly, invasive, and time consuming. Therefore, the development of cheap, fast, and easily accessible diagnostic and screening tools is needed (Humpel, 2011; Zvěrová, 2018).

At present, the most widely used tool for screening dementia is the Mini-Mental Status Examination (MMSE), which exhibits good internal consistency and concurrent validities (Boban et al., 2012; Baek et al., 2016). MMSE has been used as a clinical index to evaluate global cognitive performance with five domains: orientation, registration, attention and calculation, memory, and language (Folstein et al., 1975). Each MMSE domain functionally reflects neural activities by specific cognitive processing mechanisms. The noninvasive methods of EEG or ERPs can electrically record these neural activities. Several studies have validated the correlation between MMSE scores and EEG/ERPs variables. For instance, the study of Garn et al. (2014) explained $36 \%-51 \%$ of the variances associated with quantitative EEG markers by using MMSE scores and exhibited a strong correlation between MMSE scores and event-related potential (ERP) face-name encoding task. There was a significant negative correlation between MMSE scores with the temporal theta to alpha ratio, with $r=-0.69$ in $\mathrm{AD}$ group (Meghdadi et al., 2021). Significant correlations of MMSE with EEG beta activity were also observed (Lees et al., 2016) along with P300 latency (Tanaka et al., 1998; Lee et al., 2013). Notably, MMSE scores were effectively correlated with prefrontal EEG slowing biomarkers, as indicated from one of our previous publications (Choi et al., 2019).

Meta-analysis showed that using the MMSE alone yielded a pooled accuracy of $85 \%-87 \%$ for sensitivity and $82 \%-90 \%$ for specificity to screen dementia (cutoff value of 24-25); after adjusting for education level, the sensitivity and specificity were $97 \%$ and $70 \%$, respectively (Creavin et al., 2014). In another review of the conversion from mild cognitive impairment (MCI) into $\mathrm{AD}$ dementia, the MMSE provided $27 \%-89 \%$ pooled sensitivity with $32 \%-90 \%$ specificity (Arevalo-Rodriguez et al., 2015). Although these meta-analyses have demonstrated a moderate to high accuracy of the MMSE for screening dementia, the cross-validation approach has frequently been neglected; this has led to questions regarding the overfitting of the selected models. In the medical sciences, a cross-validation approach is being increasingly adopted to obtain an unbiased prediction accuracy with high reliability (Wong and Yeh, 2020). Even though MMSE is the most prevalent screening tool for dementia, it suffers from some limitations such as barriers due to language or educational background, the learning effect, or low sensitivity in the early stage of cognitive decline (Scazufca et al., 2009; Duff et al., 2012; Carnero-Pardo, 2014; Gross et al., 2018).

Electroencephalography (EEG) may overcome or supplement the limitations of conventional screening tools such as the MMSE for the early detection of dementia, as it is noninvasive, relatively inexpensive, and portable, while allowing repeated measurements with none or minimal learning effects (Ben-David et al., 2011). Numerous studies have demonstrated that resting-state EEG biomarkers or event-related potential 
(ERP) components obtained from EEG signals are reliable for distinguishing dementia from normal controls or other neurological disorders. For instance, by using quantitative EEG features with artificial neural networks, the classification of MCI from elderly normal individuals produced $95.87 \%$ sensitivity and 91.06\% specificity (Rossini et al., 2008) and a classification model between $\mathrm{AD}$ and MCI achieved 94.10\% accuracy (Buscema et al., 2007). Further, 92.2\% accuracy was obtained for an Area Under the Receiver Operating Characteristic curve (AUROC) of 0.965 by using the cognitive data cluster of the Consortium to Establish a Registry for the Alzheimer's Disease (CERAD) neuropsychological battery, MMSE, and clinical dementia rating; however, in combination with a quantitative EEG analysis of the absolute band power at rest, 95.3\% accuracy was achieved with an AUROC of 0.983 when distinguishing $\mathrm{AD}$ patients from non-AD persons (Fonseca et al., 2011). In addition, the N200 ERP component can identify memory changes better than MMSE (Papaliagkas et al., 2008).

With the recent advances in hardware and signal processing techniques, EEG systems with fewer channels have become emerging research topics as they can improve the simplicity and convenience of data acquisition and analysis in clinical environments. For instance, single-channel EEG signals have been tested for the detection of MCI, reaching $87.9 \%$ accuracy by using a support vector machine with leave-one-out crossvalidation (Khatun et al., 2018). Similarly, single-channel EEG features, such as the power spectrum or amplitude, and ERP features (e.g., latency) have been used to distinguish early $\mathrm{AD}$ from normal controls, while reaching $81.90 \%$ accuracy (Cho et al., 2003). More recently, Choi et al. (2019) used the prefrontal EEG signals (channels Fp1 and Fp2 in the 10-20 system) and obtained a correlation of up to 0.757 in the regression model to predict the MMSE score for older individuals.

In this study, we intended to examine whether prefrontal EEG can be used for screening dementia. First, we examined the correlations between the MMSE score and selected EEG/ERP variables. Second, we compared the distributions of selected variables between the dementia and cognitively normal persons. Third, we estimated the associations of these variables with dementia using logistic regression. Finally, we developed prediction models for dementia by combining variables from resting-state EEG, sensory event-related potential (ERP), and selective-attention ERP results. We compared the model prediction accuracies with and without the MMSE score and demographic information, and we verified the applicability of the models by performing a double cross-validation test.

\section{MATERIALS AND METHODS}

\section{Subjects}

From September to October 2017, 155 elderly individuals from four health centers (two geriatric hospitals: sites 1 and 2; two public health centers: sites 3 and 4) were recruited for this study. The participants, aged 50 years or older, were located in Uiryeong County, Korea. This observational study was performed as part of the Brain Aging Map Project, a community welfare project conducted in Uiryeong County. Four clinical research nurses were trained to operate EEG systems and other devices and performed participation scheduling, data acquisition, and result consultations. County dwellers were recruited through phone calls, brochures, flyers, and poster advertisements.

The individuals participated voluntarily for approximately, $90 \mathrm{~min}$ to measure global cognitive decline [MMSE-DS, a Korean version of the MMSE (Tae et al., 2010)], geriatric depression [KGDS, a Korean version of the Geriatric Depression Scale (GDS; Kim et al., 2001; Bae and Cho, 2004)], and EEG/ERP examinations, among others. All the confirmed demented patients were clinically diagnosed in the current centers by their clinicians or by previous medical exams conducted from other hospitals. Medical records including the diagnostic details for dementia patients were not provided in this study. Despite this limitation, dementia patients were confirmed according to a standardized diagnostic guideline, according to "Clinical practice guideline for dementia by Clinical Research Center for Dementia of South Korea” (Bon et al., 2011). This so-called CREDOS CPG was established in 2011, and offers clinical standards for AD dementia and vascular dementia in South Korea; dementia is diagnosed by comprehensive assessment of dementia, which includes historytaking, neurological examinations, neuropsychological tests, physical evaluation, brain imaging, and laboratory tests. The Diagnostic and Statistical Manual of Mental Disorders IV (DSMIV; American Psychiatric Association, 2013) was used for the dementia criteria (Bell, 1994), and the International Statistical Classification of Diseases and Related Health Problems 10th edition (ICD-10) was used to classify the disease stage (World Health Organization, 1992). All the normal individuals were recruited from public health centers with the assumption that they showed no evidence of dementia. The following individuals and subjects were excluded from the study: those who had a meal or performed intensive physical exercise within $1 \mathrm{~h}$ before beginning the experiments; those who had insufficient sleep $(<4 \mathrm{~h})$ during the previous night; those with physical abnormalities that impeded adequate EEG electrode placement; and those not apt for the study as assessed by the clinical research nurses.

Consent was obtained after providing complete descriptions about the purpose of the study to the participants or their caregivers. The study protocol was approved by the Institutional Review Board of the Korea Institute of Oriental Medicine (KIOM; approval number: I-1807/007-003). The study was performed in accordance with the Declaration of Helsinki. Figure 1 shows the consolidated standards of reporting trials (CONSORT) diagram corresponding to this study.

The demographic data, including age, sex, education level, comorbidities, and current treatments, were obtained from the participants. Subsequently, they underwent the MMSE-DS, KGDS, and EEG/ERP experiments.

\section{EEG/ERP Acquisition and Experiments}

The brain activity was noninvasively recorded via EEG at two prefrontal monopolar scalp electrodes (channels Fp1 and Fp2) 


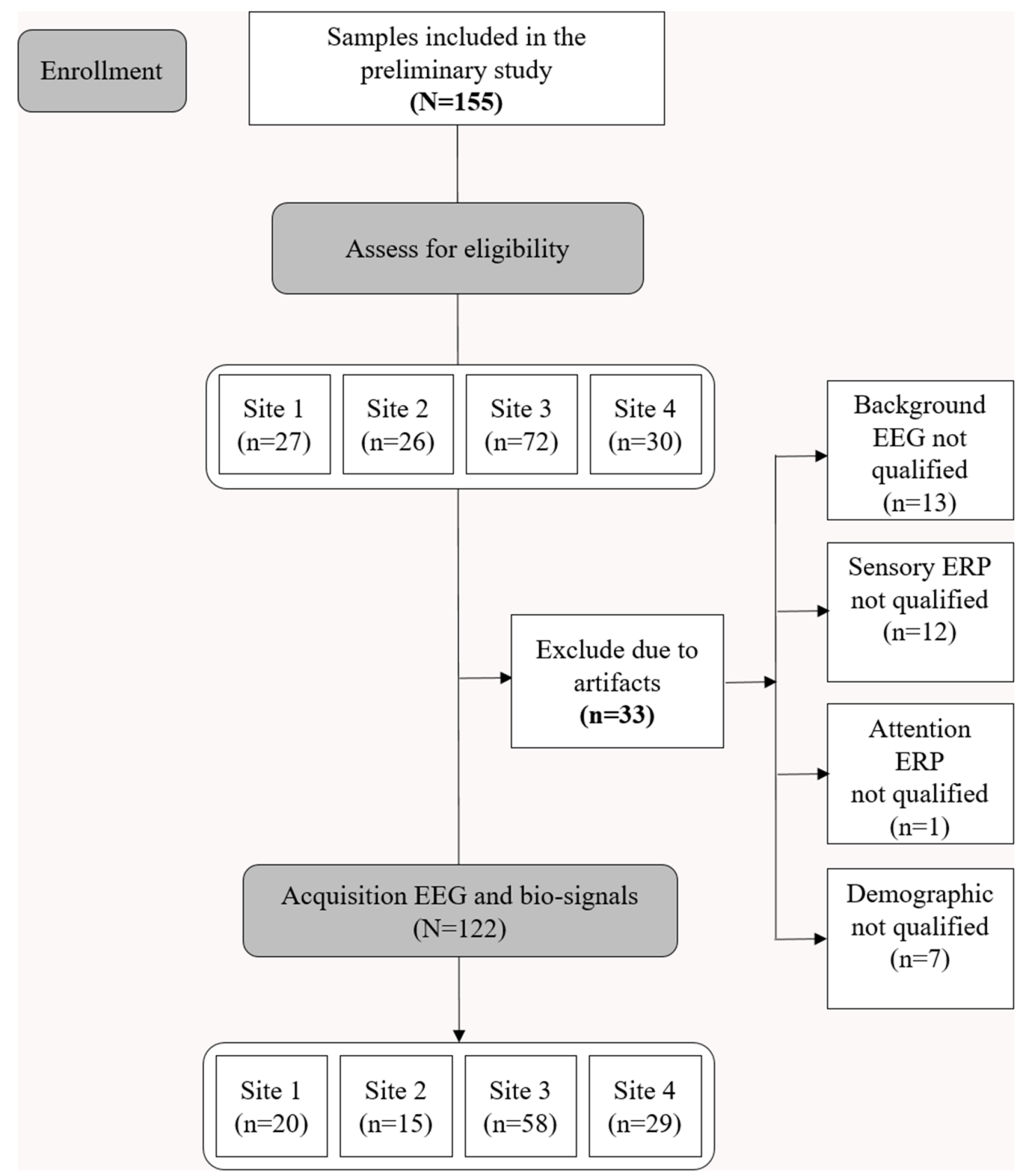

FIGURE 1 | Consolidated Standards of Reporting Trials (CONSORT) diagram illustrating enrollment and exclusion criteria for this study.

according to the International 10-20 system, with the right earlobe electrode serving as a reference. The EEG system used was the NeuroNicle FX2 (LAXTHA, Daejeon, South Korea) with band-pass filtering from 3-43 Hz and input voltages of \pm 393 $\mu \mathrm{V}$ (input noise below $0.6 \mu \mathrm{Vrms}$ ). The signals passed through an infinite impulse response, including Butterworth highpass and lowpass filters with cutoff frequencies of 2.6 and $43 \mathrm{~Hz}$, respectively. In addition, a bandstop filter was set between 55 and $65 \mathrm{~Hz}$. All the EEG electrode contact impedances were maintained below $10 \mathrm{k} \Omega$. The data were digitized in continuous recording mode at a $250 \mathrm{~Hz}$ sampling frequency and a 15-bit resolution (Choi et al., 2019). To eliminate muscle and eye movement artifacts and monitor sleepiness in the subjects, qualified operators inspected the individuals and EEG traces during the recordings. The operator guided the participants to remain comfortably seated with their eyes closed and alerted them whenever signs of behavioral or EEG drowsiness were detected. Thirty-three subjects were excluded from the study due to noise, artifacts, and incomplete demography information (Figure 1; Choi et al., 2019). 
Electroencephalography (EEG) signals from the participants were acquired while they remained seated in an upright position under three sequential conditions: (1) spontaneous brain activity to establish background EEG signals in a resting state for 5 min (resting-state EEG); (2) sensory-evoked potentials (sensory ERP) for $8 \mathrm{~min}$; and (3) a selective attention task to acquire the corresponding ERPs (selective-attention ERP) for $5 \mathrm{~min}$. All participants were tested for auditory hearing ability before operating the experiments.

To elicit the sensory ERP, each participant was instructed to avoid motion while perceiving eight intonations from auditory stimuli at $125,250,500,750,1,500,2,000,3,000$, and 4,000 $\mathrm{Hz}$. The sequence of intonation was allocated by a pseudo-random function, in which the same intonation was not provided consecutively over the 480 stimuli presented. The pseudorandomized eight intonations function as non-repeated stimuli, which helps to avoid the sensory adaptation effect and therefore maintain the response sensitivity. Sensory adaptation leads to the attenuation of neuronal responsiveness over time after the sensory neurons are exposed to a repeated stimulation (Pérez-González and Malmierca, 2014). Another reason for selecting eight intonations, lies in the fact that hearing loss due to aging generally occurs in high frequency and low frequency regimes, which would be reflected in the frequency response pattern of sensory ERP (Ciorba et al., 2011; Rigters et al., 2016). Each participant received the auditory stimuli through earphones at a volume level of $70 \mathrm{~dB}$. The duration of each stimulus was $50 \mathrm{~ms}$, with rise and fall times being within $1 \mathrm{~ms}$ and the interval between consecutive stimuli being $1 \mathrm{~s}$.

To elicit the selective-attention ERP, we adopted an active auditory oddball task presenting 64 rare random-sequenced target stimuli of 2,000 Hz (1/5 ratio) and 256 monotonic standard auditory stimuli of $750 \mathrm{~Hz}$ (4/5 ratio). The stimulus presentation was the same as that adopted to elicit the sensory ERP. The participants were asked to press a response key upon recognition of the target stimuli. The recordings were conducted while the participants kept their eyes closed in a soundless room with regular illumination.

\section{Preprocessing and Variable Extraction}

We tested data for contamination due to muscle and eye movement of the (Fp1, Fp2) prefrontal EEG signals as we did not reject any artifact in the signal processing. First, we checked that none of the EEG data were contaminated by large amounts of artifacts. Specifically, none of the participants contained more than $10 \%$ of epochs exceeding $200 \mu \mathrm{V}$ in maximum amplitude; this value was a common exclusion threshold of each epoch due to serious artifacts (Noh et al., 2006). When applying a stricter voltage threshold of $100 \mu \mathrm{V}$, we still found no participants for whom $10 \%$ of the epochs exceeded this threshold. Therefore, none of the eye-closed resting-state EEG data were rejected due to artifacts in this study.

Frequency-domain (or spectral-domain) features are typically used in the quantitative analysis of EEG rhythms. To transform an EEG signal from the time domain into the frequency domain, a Fourier transform of the autocorrelation function was employed to provide the power spectral density. In the eye-closed resting EEG, intrinsic oscillation reflective of an idling cortical state becomes dominant, and the dominant peak frequency is usually located in the $4-13 \mathrm{~Hz}$ band. Previous reports have commonly revealed that the dominant oscillatory frequencies that appear in the alpha band during normal aging become lower in cognitively disordered patients (Jackson and Snyder, 2008; Jelic and Kowalski, 2009).

Some of the variables used in the resting-state EEG results are explained further. The resting-state EEG markers were derived from a frequency-domain analysis of EEG data measured over $5 \mathrm{~min}$. Concretely, the median frequency measures the average frequency and the peak frequency measures the frequency at the maximum peak, in the dominant intrinsic oscillatory frequency band of $4-13 \mathrm{~Hz}$ of the EEG power spectrum. The alpha-to-theta ratio measures the power ratio of alpha rhythms $(8-12.99 \mathrm{~Hz})$ to theta rhythms $(4-7.99 \mathrm{~Hz})$. The EEG power spectrum was obtained by fast Fourier transform of the EEG signal using a rectangular window. The median frequency was calculated in two steps. Step 1: all spectral power values in the $4-13 \mathrm{~Hz}$ frequency domain were summed and divided by 2 . Step 2: the frequency at which the cumulative power in the $4-13 \mathrm{~Hz}$ frequency domain first, exceeded the value calculated in step 1 was selected. The peak frequency was determined as the frequency at which the power of the EEG spectrum in the $4-13 \mathrm{~Hz}$ frequency domain was largest. The absolute power was calculated in the following four frequency regions: delta $(0-3.99 \mathrm{~Hz})$, theta $(4-7.99 \mathrm{~Hz})$, alpha $(8-12.99 \mathrm{~Hz})$, and beta $(13-30 \mathrm{~Hz})$. The power data were then logarithmically transformed to fulfill the normal distributional assumptions required for parametric statistical analysis (Choi et al., 2020). The alpha-to-theta ratio was obtained by dividing the alpha power by the theta power, and the frontal asymmetry was obtained by taking the difference between the right and left alpha powers and dividing by their sum.

The ERP markers were derived from event-related potentials extracted by the conventional ensemble averaging method in EEG with stimuli. Sensory ERP variables that are exogenous sensory components represent sensory processes that mainly depend on the stimuli physical parameters and also can be influenced by cognitive processes (Pratt, 2012). The selective attention ERP components measure higher processes of cognitive function, which are related to endogenous cognitive activity (Woodman, 2010). Five variables were considered from the sensory ERP results: The average voltage peak (amplitude), average response time, amplitude deviation, response time deviation, and center-to-edge amplitude difference. Four variables were extracted from the selective-attention ERP results: the number of correct responses, response time, weighted error percentile, and voltage peak difference between the response and background ERPs. Voltage peak is the maximum amplitude of the ERP signal. The response time is the time corresponding to the voltage amplitude peak and is calculated relative to the stimulus onset. All markers were averaged over the left and right signals. The extracted variables are summarized in Table 1. 
TABLE 1 | EEG/ERP variables considered in this study.

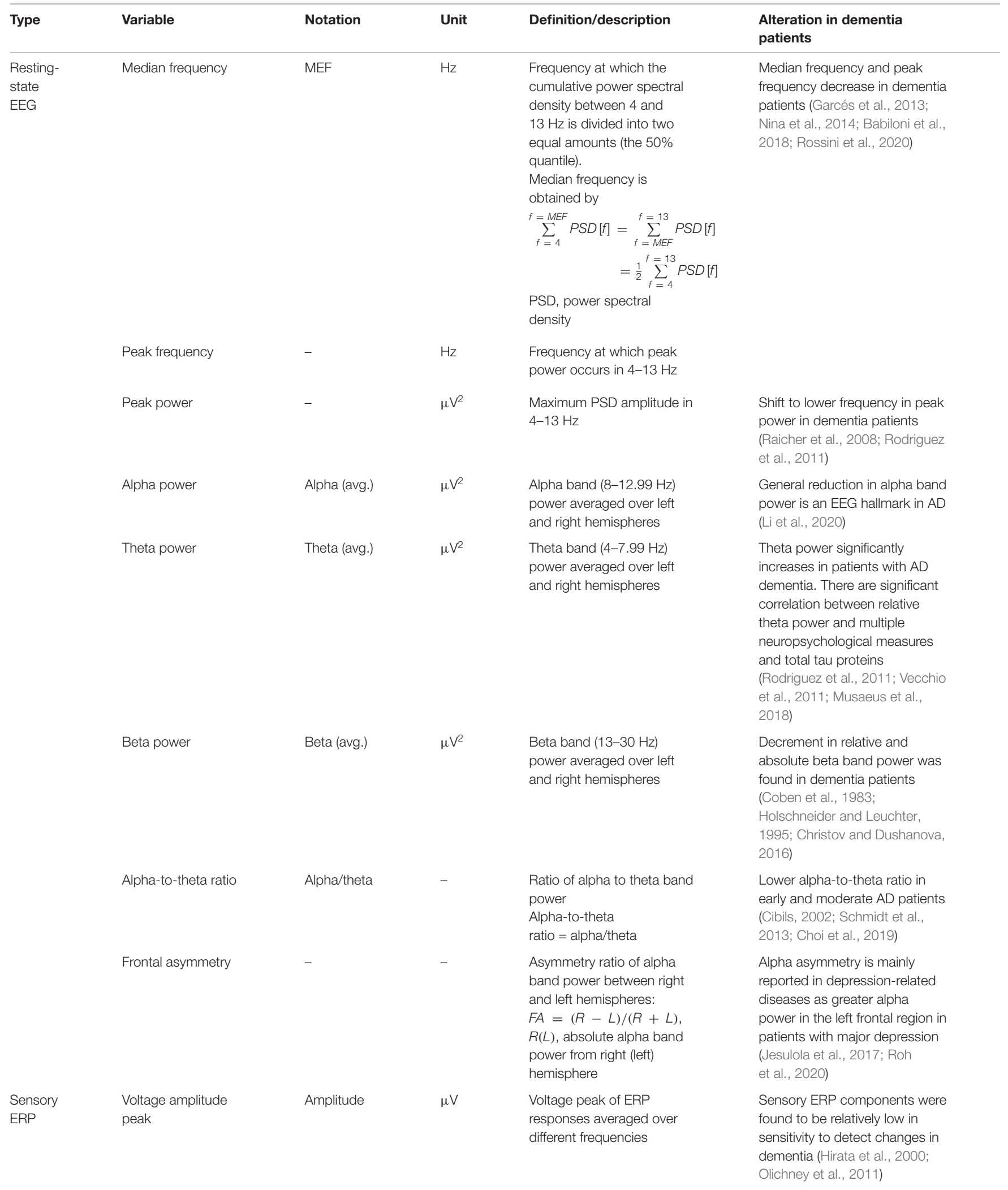


TABLE 1 | Continued

\begin{tabular}{|c|c|c|c|c|c|}
\hline Type & Variable & Notation & Unit & Definition/description & $\begin{array}{l}\text { Alteration in dementia } \\
\text { patients }\end{array}$ \\
\hline & Response time & - & $\mathrm{ms}$ & $\begin{array}{l}\text { Mean time delay between stimulus and } \\
\text { response (i.e., voltage peak) averaged } \\
\text { over different frequencies }\end{array}$ & $\begin{array}{l}\text { Delayed response across } \\
\text { different auditory and visual } \\
\text { oddball tasks in dementia } \\
\text { patients (Cecchi et al., } \\
\text { 2015; Gu et al., 2018) }\end{array}$ \\
\hline & $\begin{array}{l}\text { Voltage amplitude } \\
\text { deviation }\end{array}$ & $\begin{array}{l}\text { Amplitude } \\
\text { (deviation) }\end{array}$ & $\mu \mathrm{V}$ & $\begin{array}{l}\text { Standard deviation between voltage } \\
\text { peaks over different frequencies }\end{array}$ & - \\
\hline & $\begin{array}{l}\text { Center-to-edge } \\
\text { amplitude } \\
\text { difference }\end{array}$ & $\begin{array}{l}\text { Amplitude } \\
\text { (edge- } \\
\text { center } \\
\text { ratio) }\end{array}$ & - & $\begin{array}{l}\text { Mean voltage peaks at } 500,750, \\
1,500 \text {, and } 2,000 \mathrm{~Hz} \text { minus mean } \\
\text { voltage peaks at } 125 \text { and } 4,000 \mathrm{~Hz} \\
\text { divided by their sum }\end{array}$ & - \\
\hline & Response time & Resp. Time & s & $\begin{array}{l}\text { Time between auditory stimulation and } \\
\text { voltage peak of EEG voltage oscillations }\end{array}$ & $\begin{array}{l}\text { Response time to evoked } \\
\text { auditory stimuli increases in } \\
\text { dementia patients (Yener } \\
\text { and Başar, 2010; Gu et al., } \\
\text { 2018) }\end{array}$ \\
\hline & $\begin{array}{l}\text { Weighted error } \\
\text { percentile }\end{array}$ & wER & - & $\begin{array}{l}\text { WER }=(\text { no. errors }+4 \times(64-\text { no. } \\
\text { correct recognitions }) /(256+64 \times 4) \\
\text { No. of target (background) stimuli }=64 \\
(256)\end{array}$ & - \\
\hline & $\begin{array}{l}\text { Amplitude } \\
\text { difference between } \\
\text { response and } \\
\text { background ERP }\end{array}$ & $\begin{array}{l}\text { Amp (resp) } \\
- \text { Amp (bg) }\end{array}$ & $\mu \mathrm{V}$ & $\begin{array}{l}\text { Difference in voltage peaks of EEG } \\
\text { oscillations between target and } \\
\text { background stimuli }\end{array}$ & $\begin{array}{l}\text { Patients with AD dementia } \\
\text { showed lower amplitude for } \\
\text { ERP features (Vecchio and } \\
\text { Määttä, 2011; Cecchi et al., } \\
\text { 2015) }\end{array}$ \\
\hline
\end{tabular}

Eight variables from resting-state $E E G$, five from sensory ERP, and four from selective attention ERP. EEG, electroencephalography; ERP, event-related potential.

\section{Statistical Analysis}

The significant level for all statistical tests is set to $\alpha=0.05$. The demographic and neuropsychological characteristics were summarized as the means and standard deviations (SDs) or medians and ranges (from minimum to maximum values) for continuous variables, and as the frequencies and proportions for categorical variables for the dementia and non-dementia groups. Either an independent two-sample $t$-test or a MannWhitney-Wilcoxon rank-sum test was performed after checking the normality of each group of data based on the Shapiro-Wilk test to assess the differences in the continuous variables across dementia and non-dementia individuals. The chi-squared $\left(\chi^{2}\right)$ test or the Fisher's exact test was used to check the independence of the categorical variables from the dementia status. The association between the MMSE score and each EEG/ERP variable was evaluated using the Pearson's correlation coefficient $(\hat{\rho})$ and slope of each EEG measurement $(\hat{\beta})$ obtained from univariate linear regression analysis.

The distribution of every EEG/ERP variable for the dementia and non-dementia individuals was obtained using kernel density estimation to visualize the natural differences in both groups for illustrative purposes. Univariate and multiple logistic regression analyses were conducted to estimate the unadjusted or adjusted odds ratios for dementia in each EEG/ERP variable to assess the associations between the EEG/ERP variables and dementia prevalence. In the multiple logistic regression analysis, sex, age, education level, and GDS score were used as covariates. The underlying diseases of the participants described in Table 2 were not considered as covariates due to the small sample size. Furthermore, the MMSE score was included as an additional covariate in the regression model to identify the independent association of the EEG/ERP variables for dementia.

Dementia prediction models were developed based on all EEG/ERP and demographic variables (age, sex, and education level) that are directly associated with cognitive status. The MMSE score was also used as a single predictor to compare the performances of the models using EEG/ERP features or to investigate the improvement of the predictive models using EEG/ERP features in combination with the MMSE score. All continuous predictors were standardized to a mean of 0 and SD of 1 for data preprocessing. For the model comparisons, we generated 12 datasets based on combination of the variable groups: MMSE score, demographics, restingstate EEG, sensory ERP, and selective attention ERP. The 
TABLE 2 | Demographic information and neuropsychological test results of dementia and non-dementia subjects.

\begin{tabular}{|c|c|c|c|c|}
\hline & Total $(n=122)$ & No $(n=87)$ & Yes $(n=35)$ & Test Statistic \\
\hline \multicolumn{5}{|l|}{ Age [years] } \\
\hline Mean (SD) & $71.0( \pm 11.9)$ & $68.2( \pm 11.2)$ & $78.1( \pm 10.7)$ & \\
\hline Median [range] & 73.9 [42.3-95.9] & 68.7 [48.3-90.6] & 78.6 [42.3-95.9] & $W=777.0, p=0.0000$ \\
\hline \multicolumn{5}{|l|}{ Sex } \\
\hline Male & $30(25 \%)$ & $22(25 \%)$ & $8(23 \%)$ & \\
\hline Female & 92 (75\%) & $65(75 \%)$ & 27 (77\%) & $\chi_{(d f=1)}^{2}=0.0, p=9.605 \mathrm{E}-1$ \\
\hline \multicolumn{5}{|c|}{ Education level [year] } \\
\hline Mean (SD) & $6.0( \pm 5.0)$ & $7.1( \pm 5.0)$ & $3.4( \pm 3.9)$ & $W=2,148.5, p=0.0003$ \\
\hline Median [range] & $6.0[0.0-18.0]$ & $6.0[0.0-18.0]$ & $0.0[0.0-12.0]$ & \\
\hline \multicolumn{5}{|c|}{ Systolic BP [mmHg] } \\
\hline Mean (SD) & $125.6( \pm 16.4)$ & $125.1( \pm 15.5)$ & $126.9( \pm 18.6)$ & $t_{120.0}=-0.6, p=0.5716$ \\
\hline Median [range] & 123.5 [80.0-170.0] & $123.0[80.0-170.0]$ & 130.0 [98.0-169.0] & \\
\hline \multicolumn{5}{|c|}{ Diastolic BP [mmHg] } \\
\hline Mean (SD) & $73.5( \pm 11.7)$ & $74.3( \pm 12.0)$ & $71.4( \pm 10.8)$ & $t_{120.0}=1.3, p=0.2091$ \\
\hline Median [range] & 70.0 [41.0-100.0] & $73.0[41.0-100.0]$ & 70.0 [45.0-95.0] & \\
\hline \multicolumn{5}{|l|}{ MMSE score } \\
\hline Mean (SD) & $23.2( \pm 5.7)$ & $25.3( \pm 4.6)$ & $18.0( \pm 5.0)$ & $W=2630.0, p<1 \mathrm{E}-6$ \\
\hline Median [range] & $25.0[5.0-30.0]$ & 27.0- [12.0-30.0] & $18.0[5.0-28.0]$ & \\
\hline \multicolumn{5}{|l|}{ GDS score } \\
\hline Mean (SD) & $12.2( \pm 6.5)$ & $10.7( \pm 6.0)$ & $16.0( \pm 6.1)$ & $W=810.5, p=0.0001$ \\
\hline Median [range] & $11.0[1.0-28.0]$ & $9.0[1.0-24.0]$ & $16.0[2.0-28.0]$ & \\
\hline \multicolumn{5}{|l|}{ Diabetes } \\
\hline No & $101(83 \%)$ & $73(84 \%)$ & $28(80 \%)$ & $\chi_{(d f=1)}^{2}=0.1, p=0.8010$ \\
\hline Yes & $21(17 \%)$ & $14(16 \%)$ & $7(20 \%)$ & \\
\hline \multicolumn{5}{|l|}{ Hypertension } \\
\hline No & $60(49 \%)$ & $46(53 \%)$ & $14(40 \%)$ & $\chi_{(d f=1)}^{2}=1.2, p=0.2774$ \\
\hline Yes & $62(51 \%)$ & $41(47 \%)$ & $21(60 \%)$ & \\
\hline \multicolumn{5}{|l|}{ Hyperlipidemia } \\
\hline No & $105(86 \%)$ & 73 (84\%) & 32 (91\%) & FE-test, $p=0.3900$ \\
\hline Yes & $17(14 \%)$ & $14(16 \%)$ & $3(9 \%)$ & \\
\hline \multicolumn{5}{|l|}{ Thyroid disease } \\
\hline No & $115(94 \%)$ & $81(93 \%)$ & 34 (97\%) & FE-test, $p=0.6718$ \\
\hline Yes & $7(6 \%)$ & $6(7 \%)$ & 1 (3\%) & \\
\hline \multicolumn{5}{|l|}{ Mental disorder } \\
\hline No & $93(76 \%)$ & $81(93 \%)$ & 12 (34\%) & $\chi_{(d f=1)}^{2}=44.5, p<1 \mathrm{E}-6$ \\
\hline Yes & $29(24 \%)$ & $6(7 \%)$ & $23(66 \%)$ & \\
\hline \multicolumn{5}{|c|}{ Nervous system disease } \\
\hline No & $111(91 \%)$ & 79 (91\%) & 32 (91\%) & FE-test, $p=1.0000$ \\
\hline Yes & $11(9 \%)$ & $8(9 \%)$ & $3(9 \%)$ & \\
\hline \multicolumn{5}{|c|}{ Circulatory disease } \\
\hline No & $118(97 \%)$ & 84 (97\%) & 34 (97\%) & FE-test, $p=1.0000$ \\
\hline Yes & $4(3 \%)$ & $3(3 \%)$ & $1(3 \%)$ & \\
\hline
\end{tabular}

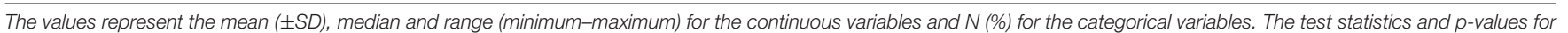

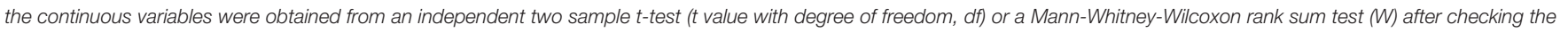

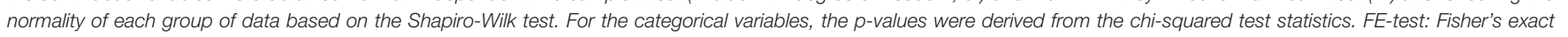
test.

interaction terms between sex and other variables were included as predictors in each candidate model containing demographic features.

In total, 122 participants were randomly split, with $80 \%$ being in the training set $(n=98)$ and $20 \%$ in the test set $(n=24)$. The dementia cases in both the training and test sets were distributed proportionally to the total sample size. Before assigning data to the training and test sets, the total dataset was stratified by the dementia status. Consequently, $20 \%$ of the data were randomly selected according to each stratum, and then the selected data from both strata were merged into the test dataset. The rest $80 \%$ of the data of both strata were merged into the training set. We trained several learning algorithms using a five-fold crossvalidation approach, for which the training dataset was again stratified according to the dementia status; subsequently, the randomly generated fold identifiers were given to each stratified group. The learning algorithms employed in this study included binary logistic regression with stepwise variable selection based on Akaike information criteria; penalized logistic regression including ridge, elastic net, and least absolute shrinkage selection operator (Friedman et al., 2020); random forest algorithm (Wright et al., 2020); and extreme gradient boosting (Chen et al., 2020). The model performance was evaluated using the AUROC and binomial deviance. The optimal model (with the highest AUROC and lowest binomial deviance) was selected within each combination of learning algorithms and 12 datasets, and its prediction power was evaluated with the test set. All statistical analyses and predictive model development were conducted using the statistical software R (version 4.0.2, released 2019-0622; R Core Team, 2020). 


\section{RESULTS}

"Subject Characteristics" section describes the basic characteristics of the participants with regard to their demographics, neuropsychological information, and comorbidities. "Correlation Between MMSE Score and EEG Measures" section demonstrates the correlation between the EEG/ERP variables and conventional MMSE scores for screening dementia using linear regression analysis. "Densities of EEG/ERP Variables Between Dementia and Non-dementia Subjects" section reports the distribution of each EEG/ERP variable by its density in the dementia and non-dementia groups. "Relation Between EEG/ERP Variables and Dementia" section clarifies the relations between the EEG/ERP variables and dementia, using the estimated odds ratios in the unadjusted and the two adjusted models based on logistic regression. Finally, "Prediction Models for Dementia" section provides an evaluation of the various dementia prediction models based on the EEG/ERP variables, MMSE scores, and demographic data.

\section{Subject Characteristics}

The overall demographic information, neuropsychological characteristics, and comorbidities of the 122 persons enrolled in this study are listed in Table 2. Among the participants, 87 were non-dementia individuals, and the remaining 35 were confirmed dementia patients. Further, 25\% of the participants were male and the remaining $75 \%$ were female. The ages of the dementia and non-dementia groups were $78.1 \pm 10.7$ and $68.2 \pm 11.2$ years (mean \pm standard deviation), respectively, and their education levels were $3.4 \pm 3.9$ and $7.1 \pm 5.0$ years $(\mathrm{p}<0.05)$, respectively. The MMSE score was $18.0 \pm 5.0$ for the dementia patients and $25.3 \pm 4.6$ for the non-dementia individuals and ranged from 5.0-30.0 $(\mathrm{p}<0.05)$. Thus, the dementia patients exhibited lower MMSE scores and education levels and higher mean ages than the non-dementia individuals (Pedraza et al., 2013; Qin et al., 2020). Moreover, the GDS score was higher in the dementia individuals ( $16.0 \pm 6.1$, mean \pm standard deviation) than in the non-dementia subjects $(10.8 \pm 6.2)$. The physiological and psychological information, such as blood pressure, diabetes, hypertension, and mental disorders, showed no statistically significant differences.

\section{Correlation Between MMSE Score and EEG Measures}

We investigated the relations between the MMSE score and prospective EEG/ERP variables from the resting-state EEG, sensory ERP, and selective-attention ERP using linear regression models and the Pearson correlation coefficients, obtaining the results shown in Figure 2. Weak to moderate linear correlations are observed between the MMSE score and EEG/ERP variables.

Among the resting-state EEG variables, the median frequency, peak frequency, and alpha-to-theta ratio show positive moderate linear correlations with the MMSE score, with average Pearson correlation coefficient $(\hat{\rho})$ of $0.55-0.68$ and average regression coefficients $(\hat{\beta})$ of $2.55-10.29$. The theta power shows a negative linear correlation with the MMSE score, with $\hat{\rho}=-0.43$ and $\hat{\beta}=-4.34$. Individual variables from the sensory ERP show weak negative correlations with the MMSE scores, with $\hat{\rho}$ from -0.12 to -0.24 . For the selective-attention ERP variables, the MMSE scores show moderate linear correlations with the most variables, including positive correlations with the number of correct responses and amplitude difference between responses, with $\hat{\rho}=0.58$ and $\hat{\rho}=0.27$, respectively, and negative correlations with the response time and weighted error percentile, with $\hat{\rho}$ ranging from -0.40 to -0.68 .

\section{Densities of EEG/ERP Variables Between Dementia and Non-dementia Subjects}

We determined the distribution of each EEG/ERP variable based on its density in the dementia and non-dementia groups, obtaining the results shown in Figure 3. Overlapping distributions are observed for some variables obtained from the resting-state EEG and sensory ERP results. However, the variables exhibiting moderate correlations with the MMSE score (reported in "Correlation Between MMSE Score and EEG Measures" section) consistently show significant differences between the dementia and non-dementia groups. Specifically, the median frequency, peak frequency, alpha-to-theta ratio, and theta power from the resting-state EEG results; average response time from the sensory ERP results; and all selectiveattention ERP variables exhibit significant differences between the dementia and non-dementia groups. Overall, the observed differences in the distributions of the EEG/ERP variables reflect the different cognitive statuses of the dementia and non-dementia groups.

\section{Relation Between EEG/ERP Variables and Dementia}

We obtained the forest plots shown in Figure 4 for the estimated odds ratios and the $95 \%$ confidence intervals of the EEG/ERP variables for predicting dementia. Three logistic regression models were considered, namely, the unadjusted model (first model); the first model adjusted for sex, age, education level, and GDS score (second model); and the second model also adjusted for the MMSE score (third model).

In the first model, most variables from the resting-state EEG and selective-attention ERP reflect the risk of dementia, with odds ratios and $95 \%$ confidence intervals, significantly different from 1. Specifically, small peak frequency, median frequency, alpha-to-theta ratio, frontal asymmetry, and large theta band power in the resting-state EEG results indicate increased risk of dementia with mean odds ratios of $0.255,0.285,0.289,0.546$, and 1.699 ( $p$-values from 1.09E-2 to 5.58E-7), respectively. Similarly, all variables from the selective-attention ERP contribute with mean odds ratios from $0.349-0.521$ and from $2.130-2.364$ ( $p$-values from $1.96 \mathrm{E}-2$ to $4.31 \mathrm{E}-5)$. In addition, the delayed average response time between the left and right hemispheres in sensory ERP also indicates increased risk of dementia with a mean odds ratio of 1.967 ( $p$-value of 1.25E-3). The detailed odds ratios and $p$-values are presented in Appendix Table A1. 


\section{A Resting State EEG}
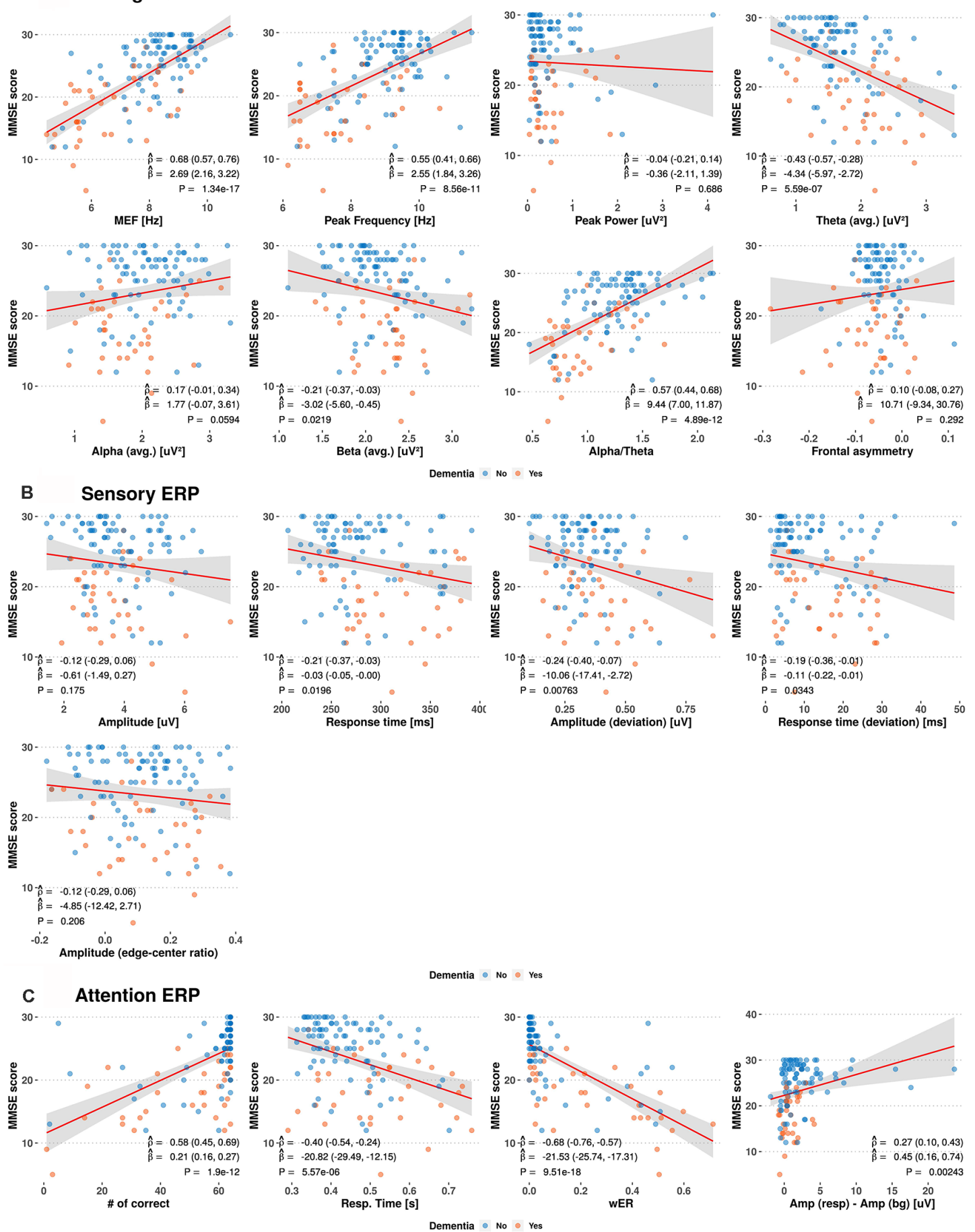

FIGURE 2 | Scatterplots between Mini-Mental Status Examination (MMSE) scores and electroencephalography (EEG)/event-related potential (ERP) variables. The red and blue circles indicate the dementia and non-dementia subjects, respectively. The red line and shaded area show the estimated regression curves and $95 \%$ confidence intervals derived from univariate regression analysis. The estimated Pearson correlation coefficient $(\hat{\rho})$, regression coefficient $(\hat{\beta})$, and $p$-value $(P)$ for each EEG/ERP variable are shown with their 95\% confidence intervals (MEF, median frequency; wER, weighted error percentile).

In the second model, only the median frequency, peak frequency, alpha-to-theta ratio, and frontal asymmetry in the resting-state EEG results and the average response time in the sensory ERP results are effective to identify dementia after adjustment, with odds ratios and $95 \%$ confidence intervals different from 1 . Notably, the bounds of the $95 \%$ confidence 


\section{A Resting State EEG}

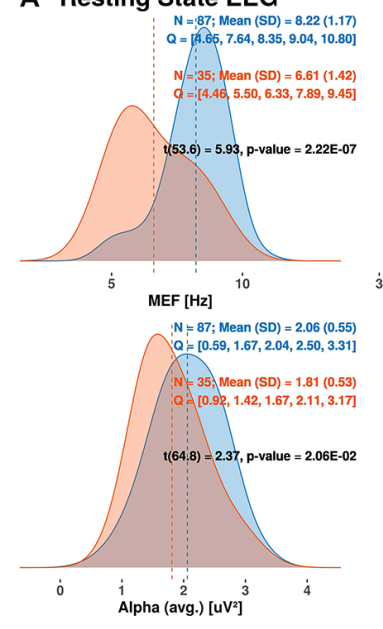

B Sensory ERP

B Sensory ERP
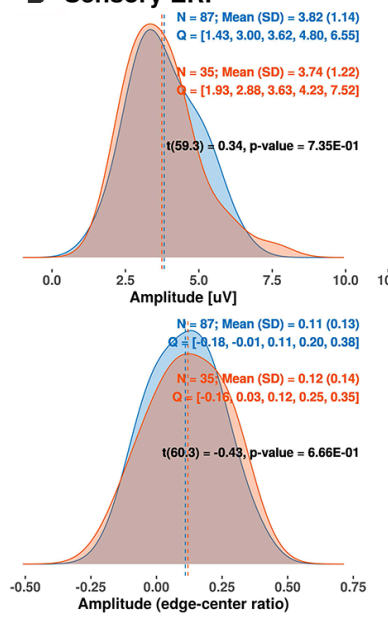

\section{Attention ERP}

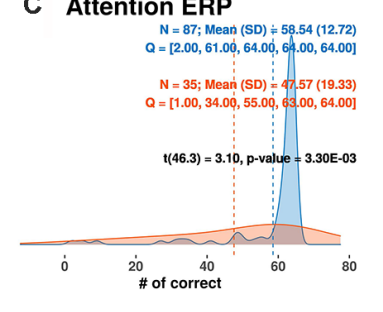

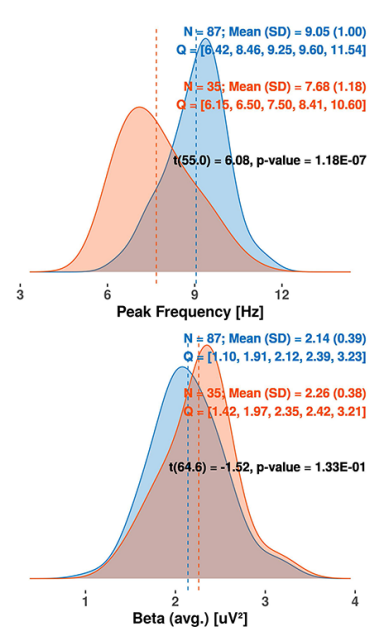
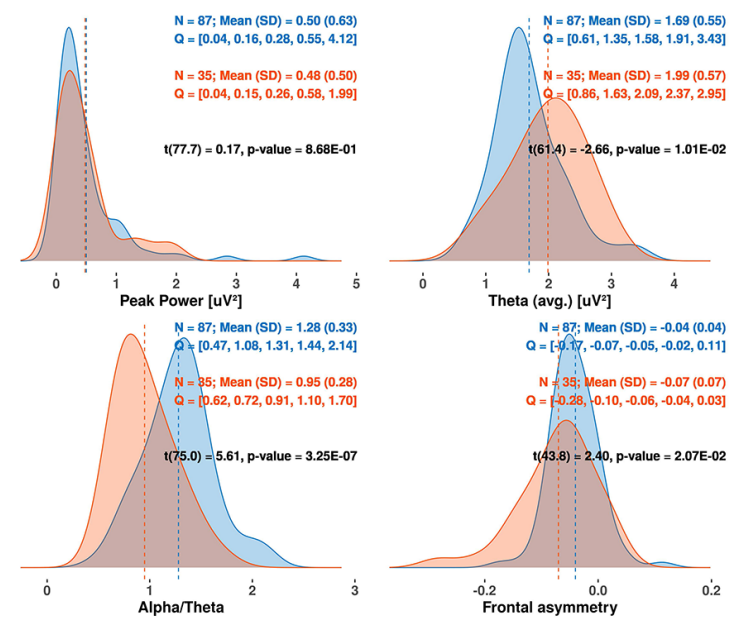

Dementia $\square$ No $\square$ res
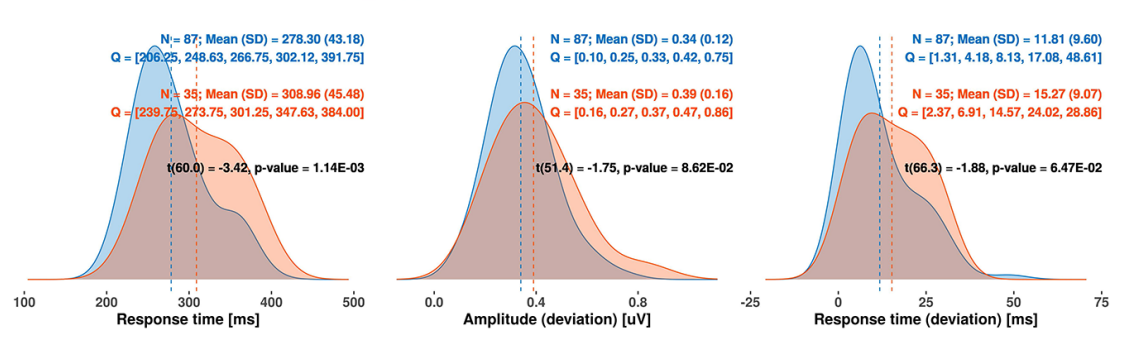
Dementia $\square$ No $\square$ Yes
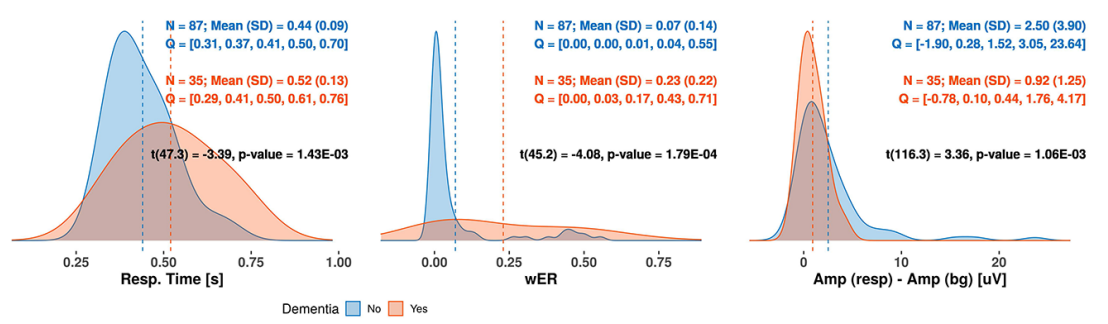

FIGURE 3 | Estimated densities of EEG/ERP variables in dementia and non-dementia subjects. Q indicates values divided into four quantiles (MEF, median frequency; wER, weighted error percentile; SD, standard deviation).

intervals of these variables in the second model are wider than those in the first model, but they still reflect the risk factors of dementia with $p<0.05$. Hence, the median frequency, peak frequency, alpha-to-theta ratio, frontal asymmetry and average response time tend to be independent from the demographic risk factors and may represent risk factors of dementia.

In the third model, most variables correlated with the MMSE score no longer represent risk factors, and only a few variables, including the peak frequency and frontal asymmetry in the resting-state EEG results and average response time in the sensory ERP results enable identification of dementia. Interestingly, frontal asymmetry shows no correlation with the MMSE score, but it represents a considerable risk factor for dementia after adjustment for demographic covariates and the MMSE score.

Variables such as the median frequency, peak frequency, and alpha-to-theta ratio in the resting-state EEG results 


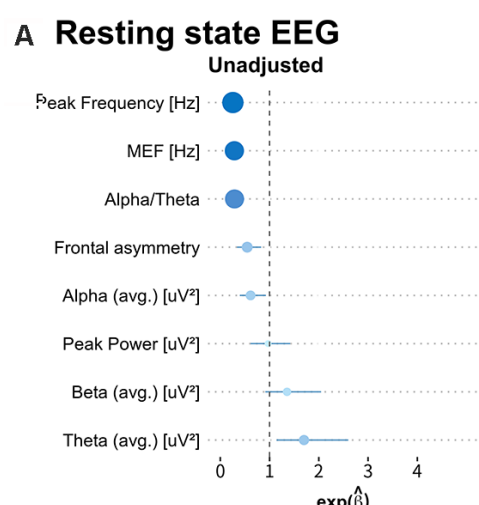

B Sensory ERP

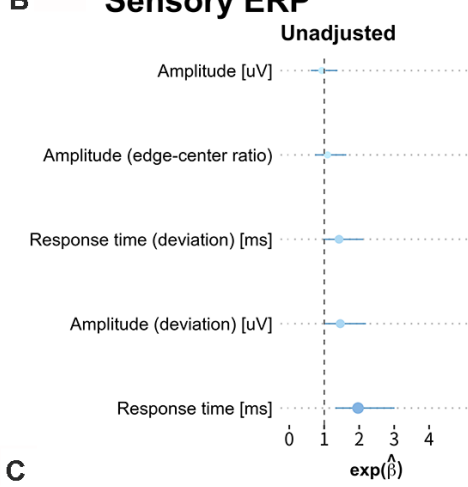

Attention ERP

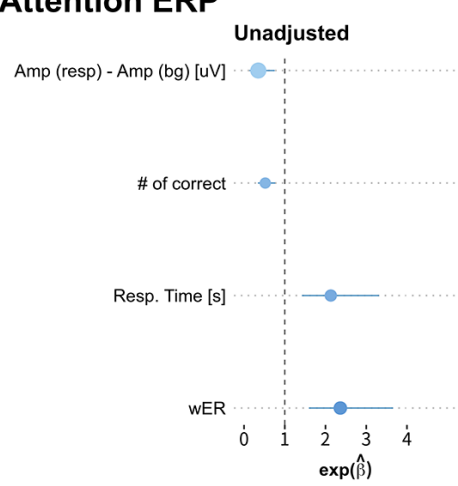

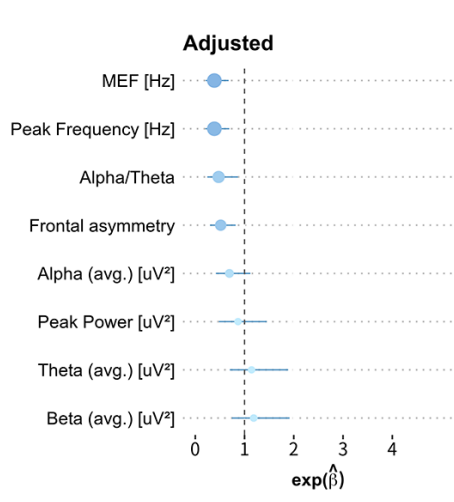
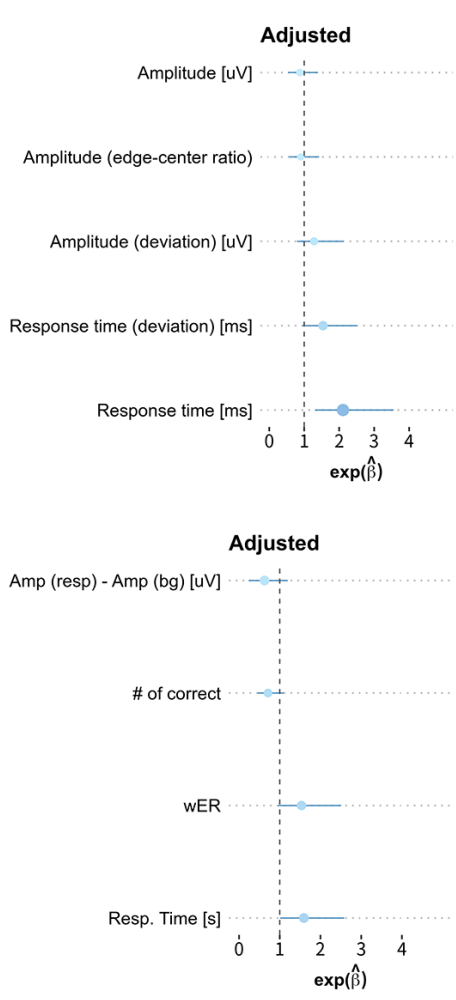
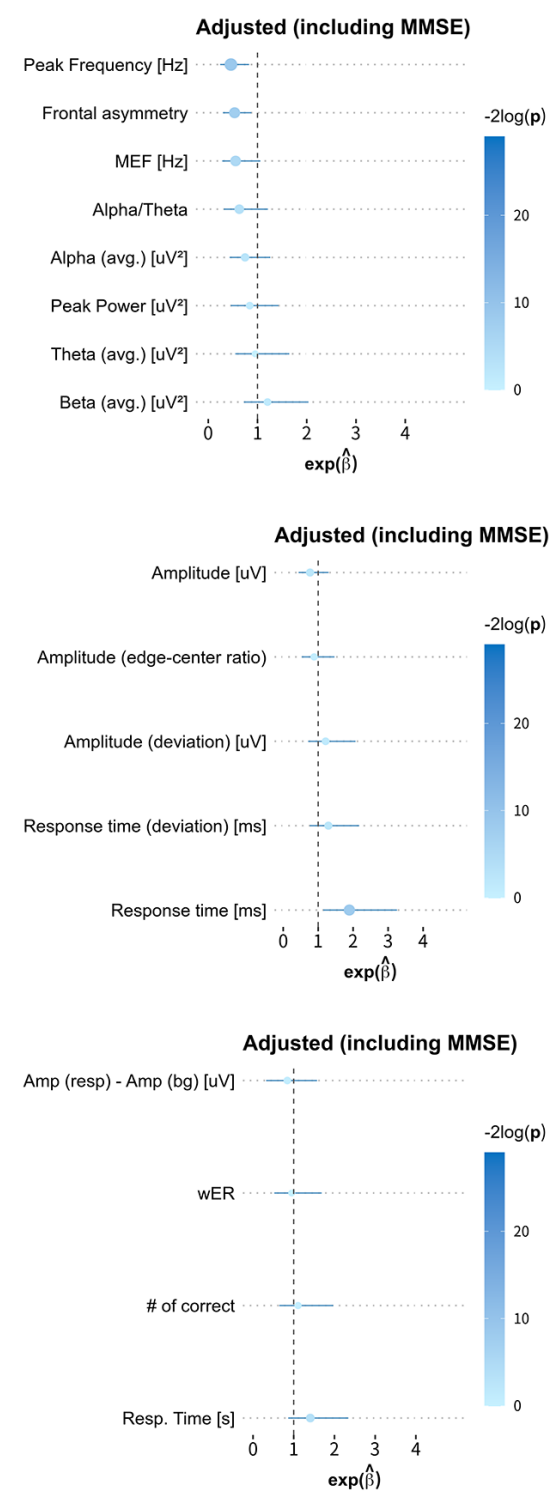

FIGURE 4 | Estimated odds ratios and 95\% confidence intervals derived from three logistic regression models. The first, second, and third columns show the results from the unadjusted univariate logistic regression model; the model adjusted for sex, age, education level, and geriatric depression scale (GDS) score; and the model adjusted for all these covariates and the MMSE score, respectively. The size of each circle indicates the magnitude of the estimated odds ratio, and the line across the circle represents its 95\% confidence interval. The color map represents the magnitude of the log-transformed $p$-values (-2log $\mathrm{p}$ ) for the odds ratios (MEF, median frequency; wER, weighted error percentile). The quantitative results are summarized in Appendix Table A1.

present moderate to strong correlations with the MMSE score (reported in "Correlation Between MMSE Score and EEG Measures" section) and provide consistent odds ratio values for classifying dementia. On the other hand, the frontal asymmetry in the resting-state EEG results and the average response times from the left and right hemispheres in the sensory ERP results show no or weak correlations with the MMSE score. Nevertheless, they exhibit valid odd ratios for classifying dementia, suggesting that they could be candidate biomarkers for dementia screening independently from the MMSE score.

\section{Prediction Models for Dementia}

We categorized the prediction model results into five groups (Table 3). The first group contained univariate analysis of MMSE, multivariate analysis of individual sets of MMSE plus demographic information, resting-state EEG, sensory ERP, and selective-attention ERP. The logistic regression model using the ordinary least squares approach for parameter estimation predicted dementia using only the MMSE score, achieving a 0.803 AUROC and 23.845 deviance. Adding demographic information to the MMSE score did not improve the accuracy. In fact, the prediction model based on logistic regression plus 
TABLE 3 | Evaluation results of prediction models according to type of data and classification model.

\begin{tabular}{|c|c|c|c|c|c|c|c|c|}
\hline & \multicolumn{2}{|c|}{$\begin{array}{c}\text { Logistic regression } \\
\text { (Ordinary least square) }\end{array}$} & \multicolumn{2}{|c|}{$\begin{array}{l}\text { Logistic regression } \\
+ \text { Elastic net }\end{array}$} & \multicolumn{2}{|c|}{ Random forest } & \multicolumn{2}{|c|}{$\begin{array}{l}\text { Extreme gradient } \\
\text { boosting }\end{array}$} \\
\hline & AUROC & Deviance & AUROC & Deviance & AUROC & Deviance & AUROC & Deviance \\
\hline MMSE & 0.803 & 23.845 & - & - & - & - & - & - \\
\hline $\mathrm{DM}+\mathrm{MMSE}$ & 0.664 & 31.380 & 0.803 & 25.234 & 0.748 & 26.200 & 0.752 & 26.995 \\
\hline RSEEG & 0.824 & 23.037 & 0.824 & 22.183 & 0.773 & 23.843 & 0.807 & 23.929 \\
\hline sensERP & 0.697 & 30.332 & 0.647 & 28.832 & 0.605 & 28.465 & 0.500 & 28.979 \\
\hline attERP & 0.891 & 20.363 & 0.882 & 21.608 & 0.857 & 20.679 & 0.882 & 24.134 \\
\hline RSEEG + sensERP + attERP & 0.739 & 42.722 & 0.849 & 21.146 & 0.832 & 22.569 & 0.849 & 21.193 \\
\hline $\mathrm{DM}+\mathrm{RSEEG}+$ sensERP + attERP & 0.571 & 73.514 & 0.832 & 21.439 & 0.832 & 21.954 & 0.832 & 21.295 \\
\hline MMSE + RSEEG & 0.798 & 25.300 & 0.849 & 22.141 & 0.807 & 21.581 & 0.849 & 22.123 \\
\hline MMSE + sensERP & 0.807 & 25.795 & 0.798 & 23.338 & 0.790 & 22.598 & 0.756 & 29.949 \\
\hline MMSE + attERP & 0.803 & 23.845 & 0.849 & 23.064 & 0.798 & 24.330 & 0.739 & 26.390 \\
\hline MMSE + RSEEG + sensERP + attERP & 0.782 & 40.182 & 0.866 & 20.855 & 0.866 & 20.875 & 0.866 & 20.986 \\
\hline $\mathrm{DM}+\mathrm{MMSE}+\mathrm{RSEEG}+$ sensERP + attERP & 0.605 & 86.032 & 0.849 & 22.048 & 0.866 & 21.140 & 0.874 & 21.150 \\
\hline Significant-variables & 0.874 & 20.628 & 0.891 & 19.397 & 0.798 & 22.908 & 0.874 & 20.461 \\
\hline
\end{tabular}

The MMSE score, demographic information (i.e., sex, age, education level, interaction between sexes, and other variables), resting-state EEG (eight variables), sensory ERP (five variables), and selective-attention ERP (four variables) were the data sources. For the models with demographic variables, the terms corresponding to the interaction between sex and other variables were included in each model. DM, Demographic information; RSEEG, resting state EEG; sensERP, sensory ERP; attERP, selective-attention ERP. For models with demographic variables, the terms corresponding to the interaction between sex and other variables are included in each model. "Significant-variables" models contain MEF, peak frequency, alpha/theta, frontal asymmetry from resting state EEG, response time from sensory ERP, and number of correct responses, response time and weighted error percentile from attention ERP. The bold fonts indicate outstanding prediction accuracies.

elastic net using the MMSE score and demographic information provided equal AUROCs of 0.803 with deviances of 25.234. In this first group, the predictor based on selective-attention ERP variables yielded the highest AUROC of 0.891 and lowest deviance of 20.363 using logistic regression. In addition, the resting-state EEG variables enabled higher accuracy than the MMSE score or the MMSE score combined with demographic information.

The second group combined resting-state EEG, sensory ERP, and selective-attention ERP before and after adding demographic information. Loosely speaking, these prediction models failed to improve accuracy compared with the models from the EEG/ERP variables in the first group.

The third group combined the MMSE score with different EEG/ERP variables. Combining the MMSE score with restingstate EEG or sensory ERP provided better prediction accuracy than using the EEG variables from the single groups or the MMSE score alone, reaching an AUROC of 0.849 and a deviance of 22.141 when using logistic regression and elastic net regularization. In comparison with selective-attention ERP alone, combining the MMSE score with selective-attention ERP or with all three EEG/ERP variables did not increase the prediction accuracy.

The fourth group combined demographic information with the MMSE score, resting-state EEG, sensory ERP, and selectiveattention ERP. This group provided a lower AUROC with higher deviance than the third group, in which demographic information was neglected.

Finally, the fifth group ("significant-variables" model) contained eight most likely potential markers among all the variables, including $\mathrm{MEF}$, peak frequency, alpha-theta, and frontal asymmetry from resting state EEG, response time from sensory ERP, and number of correct responses, response time, and weighted error percentile from selective-attention ERP. These variables were shown to have high correlations with MMSE score ("Correlation Between MMSE Score and EEG Measures" section), less overlapping in their distribution between dementia and non-dementia groups ("Densities of EEG/ERP Variables Between Dementia and Non-dementia Subjects" section), and indicated as risk factors of dementia after adjusting for covariates ("Relation Between EEG/ERP Variables and Dementia" section). This combination provided a similar AUC of 0.891 but lower deviance of 19.397 using logistic regression with elastic net in comparison with selectiveattention ERP cluster (deviance 20.363) using logistic regression with ordinary least square. In this "significant-variables" model based on the elastic net, the accuracy went up to $92.7 \%$.

The prediction model results show that the groups of restingstate EEG and selective-attention ERP variables predict dementia better than the MMSE score. In addition, the EEG/ERP variables combined with the MMSE score further improve dementia prediction, except for selective-attention ERP, whereas adding demographic information to either the EEG/ERP variables or MMSE score does not improve the prediction accuracy. The ineffectiveness of demographic information may be due to the diversity of the participants and the small sample size. The evaluation results of the prediction models are summarized in Table 3.

\section{DISCUSSION}

In this study, spontaneous resting state EEG, sensory ERP and selective-attention ERP were used as three methods to obtain the important brain oscillations (Başar et al., 2016). Both EEG and ERP variables have been investigated as potential biomarkers to detect MCI and its progression to AD dementia, as well as to directly detect AD dementia (Herrmann and Demiralp, 2005; Uhlhaas and Singer, 2006; Jackson and Snyder, 2008). In restingstate EEG, frequency components shift from high-frequency 
bands (i.e., alpha and beta) to lower frequency bands (i.e., delta and theta), and the alterations develop gradually according to the disease severity (Jelles et al., 2008; Smailovic and Jelic, 2019). Similarly, the peak frequency, median frequency, and alpha-to-theta ratio in dementia patients drift towards lower frequencies compared with non-dementia individuals (Raicher et al., 2008; Dauwels et al., 2010; Schmidt et al., 2013). In ERP, amplitude reduction and increased latency have been reported (Başar et al., 2010), as well as reduced accuracy and increased response time in a target detection task (Cecchi et al., 2015) in dementia patients. Our findings are consistent with the results of these studies.

The MMSE has been used widely in clinical practice as an effective and sensitive test to detect and screen cognitive impairment and dementia (Benson et al., 2005; ArevaloRodriguez et al., 2015). The MMSE enables dementia detection with $92 \%$ accuracy, $78 \%-84 \%$ sensitivity, and $87 \%-91 \%$ specificity (cutoff value of 23/24) (Tsoi et al., 2015). However, the MMSE has bias according to the socio-educational backgrounds of participants, practice effect, and low sensitivity in the early stage of cognitive decline (Scazufca et al., 2009; Duff et al., 2012; Carnero-Pardo, 2014). These disadvantages can be overcome while enhancing the diagnostic accuracy by combining the MMSE score with EEG/ERP data.

Selective-attention ERP examines the cognitive performance using auditory oddball paradigm, which elicits P300 in response to the target intonations through the use of prompt button pushing. This motoric response can cause a distinct movementrelated potential, which has been reported to interfere with the topography of P300 and alter its amplitude in comparison with the silent-count task (Salisbury et al., 2001; van Vliet et al., 2014; Kim et al., 2020). Despite these reported influences on P300 with button-pushing behavior, for old participants with as many as 64 deviant stimuli, the button-press was an optimal task touse the counts of correct and erroneous responses as the two salient variables in evaluating cognitive performance.

Selective-attention ERP variables include the number of correct responses, response time, weighted error percentile, and amplitude difference between deviant and background stimuli. Selective attention ERP has been shown to provide the highest AUROC values, while demonstrating the best dementia predictor among all the possible combinations of dementia risk factors. Selective-attention ERP or attention components of P300 have been studied as indicators for cognitive processing. Selective-attention ERP endogenous components reflect the ability of cognitively processing the stimulus based on the levels of attention and arousal (Polich and Kok, 1995). A prolonged P300 response time implies that more time is required to process information, which represents an index of abnormal cognition ability (Williams et al., 1991; van Deursen et al., 2009). P300 amplitude reduction in dementia patients shows that lower attentional resources were devoted to the task performance (van Deursen et al., 2009; Hedges et al., 2016). Furthermore, decreasing number of correct answers and increasing weighted error percentile in the dementia group as compared to those in the normal group indicate a reduction in attentional maintenance and action control ability during cognitive processing throughout the task (Vecchio and Määttä, 2011). All changes in selectiveattention ERP variables indicate a decrease in intrinsic brain activation to the responses in demented patients. Selectiveattention ERP provides a sensitive and reliable measure for the early detection of cognitive impairment related to AD (Cecchi et al., 2015; Gu et al., 2018). Our findings upheld the literature associated with using attention ERP for detecting dementia.

As indicated by the significant odds ratios before and after adjusting for sex, age, education level, and GDS score, the EEG/ERP variables show high correlations with the MMSE score and indicate dementia risk factors. Furthermore, variables with low correlations with the MMSE score (e.g., frontal asymmetry in resting-state EEG) may be suitable for classifying dementia independently from the MMSE score, as indicated by the significant odds ratios that are obtained after adjusting for the covariates plus the MMSE score. Frontal asymmetry has been used as an indicator of depression due to the hyperactivity of the right prefrontal lobe and the withdrawal behavior to aversive stimuli (Thibodeau et al., 2006; Jesulola et al., 2015). However, to the best of our knowledge, frontal asymmetry has not been reported as a candidate indicator of dementia. Thus, our findings establish a new direction for research on dementia by considering frontal alpha asymmetry.

Considering dementia and its relation to depression, half of the patients with late-onset depression may exhibit cognitive impairment, and the prevalence of depression in dementia patients is between 9\% and 68\% (Muliyala and Varghese, 2010). Asymmetry in frontal cortex activity reflected in EEG signals has been described as a potential discriminator for depression, such that frontal alpha asymmetry has been found to be significantly higher in depressed subjects than healthy controls (Gollan et al., 2014; Adolph and Margraf, 2017; Brzezicka et al., 2017); however, contradicting results have also been reported (van der Vinne et al., 2017; Kaiser et al., 2018). Our results may suggest that the frontal alpha asymmetry as one of the potential EEG variables for dementia detection.

We derived prediction models using different combinations of EEG/ERP variables, MMSE scores, and demographic data. Selective-attention ERP variables and resting-state EEG variables produced more accurate predictions than MMSE scores or MMSE scores combined with demographic information. Hence, these variables may be representative in the identification of cognitive changes due to dementia. In contrast, adding demographic information tended to decrease the accuracy compared to the cases in which demographic information was neglected. Hence, demographic information may undermine predictive modeling of dementia.

The variable selection in the prediction model based on the statistical test often leads to serious bias in maximizing the performance of the predictive model, as explained by Lo et al. (2015). To overcome this limitation, we adopted penalized regression approaches that performed the variable selection continuously. In our case, a model with the variables that showed highest statistical significances resulted in best accuracy among various prediction models (Table 3). In particular, the model 
exhibiting the highest AUROC (0.891) and lowest deviance (19.397) employed the eight most significant-variables in the logistic regression approach with elastic net regularization, followed by the selective-attention ERP variables in a logistic regression model via the ordinary least squares method. It implies that a prediction model with only few EEG/ERP variables that showed high statistical significance can be used for effective screening of dementia, which would lead to the cost effective utility of "prefrontal EEG" in clinics.

Overall, the logistic regression model with elastic net regularization tended to perform better than the random forest or extreme gradient boosting approach in terms of AUROC and deviance from individual EEG/ERP variables with or without MMSE. Again, adding demographic information to this model reduced the predictive performance. The adverse effects of demographics may be due to the diversity of participants considered in this study regarding aspects such as age, sex, education level, GDS score, and the underlying disease causing dementia.

Some limitations of this study remain to be addressed. The dementia patients in this study were registered in the Korean National Health Insurance Service, and we were not able to obtain further medical records of the patients, such as imaging data, to identify the underlying causes and statuses of dementia. Therefore, hidden comorbidities inducing diversity of EEG/ERP features may have affected our results. In addition, our findings cannot be generalized due to the small sample size (122 participants) and discrepancies in age and education level among groups. Even though we attempted to remove confounding effects by adjusting for age, sex, education level and depression level, the prediction models could increase clinical usability if the data had no such discrepancies in other risk factors between dementia patients and normal controls. Finally, we could not examine the exposures or suspected risk factors over time. Thus, a prospective or case-control study with a larger and more representative sample is still required to clinically validate the diagnostic value of the EEG/ERP variables considered in our study.

\section{CONCLUSION}

Prefrontal EEG variables, which are related to EEG slowing, left-right asymmetry in the resting state, and sensory and selective-attention ERPs, have been correlated with the MMSE score. Logistic regression for dementia prediction shows that most of the selected variables remain significant after adjustment for GDS and demographic risk factors of dementia, such as age, education level, and sex. In contrast, when the model is adjusted for the MMSE score and demographic covariates, these prefrontal EEG variables become non-significant, except for the frontal asymmetry among the activity in the left and right hemispheres, peak frequency in resting-state EEG, and the response time in sensory ERP. The other variables have no or minimal correlations with the MMSE score after such adjustment. From multivariate regression models with five-fold cross-validation, we found that the prefrontal
EEG variables outperform the MMSE score in dementia prediction. In particular, the prediction accuracy was the highest when using the eight variables that showed highest statistical significances among tested EEG/ERP variables. Adding demographic information fails to improve the prediction accuracy. Overall, the slowing and asymmetry of prefrontal EEG activity seem promising for dementia screening, and can be used in combination with the MMSE score or function as its alternative. In a future study, the clinical usability of few-channel EEG can be improved by recruiting more participants with balanced demographic risk factors among patient and control groups and by including preceding stages of dementia such as MCI; screening MCI patients effectively allows early medical intervention that can prevent or deter the progression to dementia.

\section{DATA AVAILABILITY STATEMENT}

The original contributions presented in the study are included in the article, further inquiries can be directed to the corresponding author.

\section{ETHICS STATEMENT}

The studies involving human participants were reviewed and approved by Declaration of Helsinki. The patients/participants provided their written informed consent to participate in this study.

\section{AUTHOR CONTRIBUTIONS}

DD led the manuscript preparation. BK analyzed the data and wrote the manuscript. JC extracted relevant EEG variables and controlled the EEG data quality. MO analyzed the data. WC designed and led the Brain Aging Map Project. KK worked to obtain the institutional review board approval. JK designed the study and wrote the manuscript. All authors commented on and approved the contents of the manuscript. All authors contributed to the article and approved the submitted version.

\section{FUNDING}

This study was conducted as a part of the Brain Aging Map Project and was supported by the Korea Institute of Oriental Medicine (KIOM; Grant no.: KSN2021130) funded by the Korean government. The Brain Aging Map Project was supported by the Human Anti-Aging Standards Research Institute, the Uiryeong Community Health Center, and the KIOM.

\section{ACKNOWLEDGMENTS}

We thank all the contributors to the Brain Aging Map Project, especially for acquiring the data used in this study. The contributors include Young Gooun You, Miok Jo, Minji Kwon, Youyoung Choi, Segyeong Jung, Soyoung Ryu, and Eunjeong Park. 


\section{REFERENCES}

Adolph, D., and Margraf, J. (2017). The differential relationship between trait anxiety, depression and resting frontal $\alpha$-asymmetry. J. Neural Transm. 124, 379-386. doi: 10.1007/s00702-016-1664-9

Alzheimer's Association Report. (2020). Alzheimer's disease facts and figures. Alzheimers Dement. 16, 391-460. doi: 10.1002/alz.12068

American Psychiatric Association. (2013). Diagnostic and Statistical Manual of Mental Disorders. 4th Edn. Arlington, VA: American Psychiatric Association.

Arevalo-Rodriguez, I., Smailagic, N., Roquéi Figuls, M., Ciapponi, A., SanchezPerez, E., Giannakou, A., et al. (2015). Mini-mental state examination (MMSE) for the detection of Alzheimer's disease and other dementias in people with mild cognitive impairment (MCI). Cochrane Database Syst. Rev. 2015:CD010783. doi: 10.1002/14651858.CD010783.pub2

Babiloni, C., Del Percio, C., Lizio, R., Noce, G., Lopez, S., Soricelli, A., et al. (2018). Abnormalities of resting state cortical EEG rhythms in subjects with mild cognitive impairment due to Alzheimer's and lewy body diseases. J. Alzheimers Dis. 62, 247-268. doi: 10.3233/JAD-170703

Bae, J. N., and Cho, M. J. (2004). Development of the korean version of the geriatric depression scale and its short form among elderly psychiatric patients. J. Psychosom. Res. 57, 297-305. doi: 10.1016/j.jpsychores.2004.01.004

Baek, M. J., Kim, K., Park, Y. H., and Kim, S. Y. (2016). The validity and reliability of the mini-mental state examination-2 for detecting mild cognitive impairment and Alzheimer's disease in a korean population. PLoS One 11:e0163792. doi: 10.1371/journal.pone.0163792

Başar, E., Gölbaşı, B. T., Tülay, E., Aydın, S., and Başar-Eroğlu, C. (2016). Best method for analysis of brain oscillations in healthy subjects and neuropsychiatric diseases. Int. J. Psychophysiol. 103, 22-42. doi: 10.1016/j. ijpsycho.2015.02.017

Başar, E., Güntekin, B., Tülay, E., and Yener, G. G. (2010). Evoked and event related coherence of Alzheimer patients manifest differentiation of sensorycognitive networks. Brain Res. 1357, 79-90. doi: 10.1016/j.brainres.2010.08.054

Bell, C. C. (1994). DSM-IV: diagnostic and statistical manual of mental disorders. JAMA 272, 828-829. doi: 10.1001/jama.1994.03520100096046

Ben-David, B. M., Campeanu, S., Tremblay, K. L., and Alain, C. (2011). Auditory evoked potentials dissociate rapid perceptual learning from task repetition without learning. Psychophysiology 48, 797-807. doi: 10.1111/j.1469-8986.2010. 01139.x

Benson, A. D., Slavin, M. J., Tran, T. T., Petrella, J. R., and Doraiswamy, P. M. (2005). Screening for early Alzheimer's disease: is there still a role for the mini-mental state examination? Prim. Care Companion J. Clin. Psychiatry 7 , 62-67. doi: 10.4088/pcc.v07n0204

Boban, M., Malojčić, B., Mimica, N., Vuković, S., Zrilić, I., Hof, P. R., et al. (2012). The reliability and validity of the mini-mental state examination in the elderly croatian population. Dement. Geriatr. Cogn. Disord. 33, 385-392. doi: $10.1159 / 000339596$

Bon, D. K., Kim, S. G., Lee, J. Y., Park, K. H., Shin, J. H., Kim, K. K., et al. (2011). Clinical practice guideline for dementia by clinical research center for dementia of south korea. J. Korean Med. Assoc. 54, 861-875. doi: 10.5124/jkma.2011.54. 8.861

Brzezicka, A., Kamiński, J., Kamińska, O. K., Woyńczyk-Gmaj, D., and Sedek, G. (2017). Frontal EEG $\alpha$ band asymmetry as a predictor of reasoning deficiency in depressed people. Cogn. Emot. 31, 868-878. doi: 10.1080/02699931.2016. 1170669

Buscema, M., Capriotti, M., Bergami, F., Babiloni, C., Rossini, P., and Grossi, E. (2007). The implicit function as squashing time model: a novel parallel nonlinear EEG analysis technique distinguishing mild cognitive impairment and Alzheimer's disease subjects with high degree of accuracy. Comput. Intell. Neurosci. 2007:35021. doi: 10.1155/2007/35021

Carnero-Pardo, C. (2014). Should the mini-mental state examination be retired? Neurologia 29, 473-481. doi: 10.1016/j.nrl.2013.07.003

Cecchi, M., Moore, D. K., Sadowsky, C. H., Solomon, P. R., Doraiswamy, P. M., Smith, C. D., et al. (2015). A clinical trial to validate event-related potential markers of Alzheimer's disease in outpatient settings. Alzheimers Dement. 1, 387-394. doi: 10.1016/j.dadm.2015.08.004

Chen, T., He, T., Benesty, M., Khotilovich, V., Tang, Y., Cho, H., et al. (2020). Xgboost: Extreme Gradient Boosting. Available online at: https://CRAN.Rproject.org $/$ package $=$ xgboost.
Chertkow, H., Feldman, H. H., Jacova, C., and Massoud, F. (2013). Definitions of dementia and predementia states in Alzheimer's disease and vascular cognitive impairment: consensus from the canadian conference on diagnosis of dementia. Alzheimers Res. Ther. 5:S2. doi: 10.1186/ alzrt198

Cho, S. Y., Kim, B. Y., Park, E. H., Kim, J. W., Whang, W. W., Han, S. K., et al. (2003). "Automatic recognition of Alzheimer's disease with single channel EEG recording," in Proceedings of the 25th Annual International Conference of the IEEE Engineering in Medicine and Biology (Cancun, Mexico: IEEE), $2655-2658$.

Choi, J., Ku, B., You, Y. G., Jo, M., Kwon, M., Choi, Y., et al. (2019). Resting-state prefrontal EEG biomarkers in correlation with MMSE scores in elderly individuals. Sci. Rep. 9:10468. doi: 10.1038/s41598-01946789-2

Choi, J., Lim, E., Park, M. G., and Cha, W. (2020). Assessing the retest reliability of prefrontal EEG markers of brain rhythm slowing in the eyes-closed resting state. Clin. EEG Neurosci. 51, 348-356. doi: 10.1177/15500594209 14832

Christov, M., and Dushanova, J. (2016). Functional correlates of the aging brain: $\beta$ frequency band responses to age-related cortical changes. Int. J. Neurorehabil. 76, 98-109. doi: 10.21307/ane-2017-009

Cibils, D. (2002). "Chapter 43 Dementia and qEEG (Alzheimer's disease)," in Supplements to Clinical Neurophysiology, Vol. 54 (Elsevier Science B.V.), 289-294.

Ciorba, A., Benatti, A., Bianchini, C., Aimoni, C., Volpato, S., Bovo, R., et al. (2011). High frequency hearing loss in the elderly: effect of age and noise exposure in an Italian group. J. Laryngol. Otol. 125, 776-780. doi: $10.1017 / \mathrm{S} 0022215111001101$

Coben, L. A., Danziger, W. L., and Berg, L. (1983). Frequency analysis of the resting awake EEG in mild senile dementia of Alzheimer type. Electroencephalogr. Clin. Neurophysiol. 55, 372-380. doi: 10.1016/0013-4694(83)90124-4

Creavin, S. T., Noel-Storr, A. H., Smailagic, N., Giannakou, A., Ewins, E., Wisniewski, S., et al. (2014). Mini-mental state examination (MMSE) for the detection of Alzheimer's dementia and other dementias in asymptomatic and previously clinically unevaluated people aged over 65 years in community and primary care populations. Cochrane Database Syst. Rev. 2014:CD011145. doi: 10.1002/14651858.CD011145.pub2

Dauwels, J., Vialatte, F., and Cichocki, A. (2010). Diagnosis of Alzheimer's disease from EEG signals: where are we standing? Curr. Alzheimer Res. 999, 1-19. doi: 10.2174/1567210204558652050

Duff, K., Chelune, G., and Dennett, K. (2012). Within-session practice effects in patients referred for suspected dementia. Dement. Geriatr. Cogn. Disord. 33, 245-249. doi: $10.1159 / 000339268$

Farooqui, A. A. (2019). "Neurochemical aspects of dementia," in Molecular Mechanisms of Dementia (Elsevier), 1-38. doi: 10.1016/b978-0-12-8163474.00001-5

Folstein, M. F., Folstein, S. E., and McHugh, P. R. (1975). "Mini-mental state". A practical method for grading the cognitive state of patients for the clinician. J. Psychiatr. Res. 12, 189-198. doi: 10.1016/0022-3956(75)90026-6

Fonseca, L. C., Tedrus, G. M. A. S., Fondello, M. A., Reis, I. N., and Fontoura, D. S. (2011). EEG theta and $\alpha$ reactivity on opening the eyes in the diagnosis of Alzheimer's disease. Clin. EEG Neurosci. 42, 185-189. doi: $10.1177 / 155005941104200308$

Friedman, J., Hastie, T., Tibshirani, R., Narasimhan, B., Tay, K., and Simon, N. (2020). Glmnet: Lasso and Elastic-Net Regularized Generalized Linear Models. Available online at: https://CRAN.R-project.org/package $=$ glmnet.

Garcés, P., Vicente, R., Wibral, M., Pineda-Pardo, J. á., López, M. E., Aurtenetxe, S., et al. (2013). Brain-wide slowing of spontaneous $\alpha$ rhythms in mild cognitive impairment. Front. Aging Neurosci. 5:100. doi: 10.3389/fnagi.2013. 00100

Garn, H., Waser, M., Deistler, M., Schmidt, R., Dal-Bianco, P., Ransmayr, G., et al. (2014). Quantitative EEG in Alzheimer's disease: cognitive state, resting state and association with disease severity. Int. J. Psychophysiol. 93, 390-397. doi: 10.1016/j.ijpsycho.2014.06.003

Gollan, J. K., Hoxha, D., Chihade, D., Pflieger, M. E., Rosebrock, L., and Cacioppo, J. (2014). Frontal $\alpha$ EEG asymmetry before and after behavioral activation treatment for depression. Biol. Psychol. 99, 198-208. doi: 10.1016/j. biopsycho.2014.03.003 
Gross, A. L., Chu, N., Anderson, L., Glymour, M. M., and Jones, R. N. (2018). Do people with Alzheimer's disease improve with repeated testing? Unpacking the role of content and context in retest effects. Age Ageing 47, 866-871. doi: 10.1093/ageing/afy136

Gu, L., Chen, J., Gao, L., Shu, H., Wang, Z., Liu, D., et al. (2018). Cognitive reserve modulates attention processes in healthy elderly and amnestic mild cognitive impairment: an event-related potential study. Clin. Neurophysiol. 129, 198-207. doi: 10.1016/j.clinph.2017.10.030

Hedges, D., Janis, R., Mickelson, S., Keith, C., Bennett, D., and Brown, B. L. (2016). P300 amplitude in Alzheimer's disease: a meta-analysis and metaregression. Clin. EEG Neurosci. 47, 48-55. doi: 10.1177/15500594145 50567

Herrmann, C. S., and Demiralp, T. (2005). Human EEG $\gamma$ oscillations in neuropsychiatric disorders. Clin. Neurophysiol. 116, 2719-2733. doi: 10.1016/j. clinph.2005.07.007

Hirata, K., Hozumi, A., Tanaka, H., Kubo, J., Zeng, X. H., Yamazaki, K., et al. (2000). Abnormal information processing in dementia of Alzheimer type. A study using the event-related potential's field. Eur. Arch. Psychiatry Clin. Neurosci. 250, 152-155. doi: 10.1007/s004060070033

Holschneider, D. P., and Leuchter, A. F. (1995). $\beta$ activity in aging and dementia. Brain Topogr. 8, 169-180. doi: 10.1007/BF01199780

Humpel, C. (2011). Identifying and validating biomarkers for Alzheimer's disease. Trends Biotechnol. 29, 26-32. doi: 10.1016/j.tibtech.2010.09.007

Jack, C. R. Jr., Bennett, D. A., Blennow, K., Carrillo, M. C., Dunn, B., Budd Haeberlein, S., et al. (2018). NIA-AA research framework: toward a biological definition of Alzheimer's disease. Alzheimers Dement. 14, 535-562. doi: 10.1016/j.jalz.2018.02.018

Jackson, C. E., and Snyder, P. J. (2008). Electroencephalography and eventrelated potentials as biomarkers of mild cognitive impairment and mild Alzheimer's disease. Alzheimers Dement. 4, S137-S143. doi: 10.1016/j.jalz.2007. 10.008

Jelic, V., and Kowalski, J. (2009). Evidence-based evaluation of diagnostic accuracy of resting EEG in dementia and mild cognitive impairment. Clin. EEG Neurosci. 40, 129-142. doi: 10.1177/155005940904000211

Jelles, B., Scheltens, W. M., van der Flier, E. J., Jonkman, F. H., Lopes da Silva, F. H., and Stam, C. J. (2008). Global dynamical analysis of the EEG in Alzheimer's disease: frequency-specific changes of functional interactions. Clin. Neurophysiol. 119, 837-841. doi: 10.1016/j.clinph.2007.12.002

Jesulola, E., Sharpley, C. F., and Agnew, L. L. (2017). The effects of gender and depression severity on the association between $\alpha$ asymmetry and depression across four brain regions. Behav. Brain Res. 321, 232-239. doi: 10.1016/j.bbr. 2016.12.035

Jesulola, E., Sharpley, C. F., Bitsika, V., Agnew, L. L., and Wilson, P. (2015). Frontal $\alpha$ asymmetry as a pathway to behavioral withdrawal in depression: research findings and issues. Behav. Brain Res. 292, 56-67. doi: 10.1016/j.bbr.2015. 05.058

Kaiser, A. K., Doppelmayr, M., and Iglseder, B. (2018). $\alpha$-asymmetrie im elektroenzephalogramm bei geriatrischer depression: valide oder verschwunden? Z. Gerontol. Geriatr. 51, 200-205. doi: 10.1007/s00391016-1108-z

Khatun, S., Morshed, B. I., and Bidelman, G. M. (2018). "Single channel EEG based score generation to monitor the severity and progression of mild cognitive impairment," in IEEE International Conference on Electro Information Technology (Rochester, MI: IEEE), 882-886.

Kim, J., Lee, K., and Lee, E. (2020). N100, N200, and P300 auditory event-related potentials depending on handedness and response tasks such as button pressing and mental counting. Audiol. Speech Res. 16, 314-320. doi: 10.21848/asr. 200059

Kim, J. M., Prince, M. J., Seon Shin, I. L., and Yoon, J. S. (2001). Validity of korean form of geriatric depression scale (KGDS) among cognitively impaired koren elderly and development of a 15-item short version (KGDS-15). Int. J. Methods Psychiatr. Res. 10, 204-210. doi: 10.1002/mpr.117

Kocahan, S., and Doan, Z. (2017). Mechanisms of Alzheimer's disease pathogenesis and prevention: the brain, neural pathology, N-methyl-Daspartate receptors, tau protein and other risk factors. Clin. Psychopharmacol. Neurosci. 15, 1-8. doi: 10.9758/cpn.2017.15.1.1

Lee, M.-S., Lee, S.-H., Moon, E.-O., Moon, Y.-J., Kim, S., Kim, S.-H., et al. (2013). Neuropsychological correlates of the P300 in patients with Alzheimer's disease.
Prog. Neuropsychopharmacol. Biol. Psychiatry 40, 62-69. doi: 10.1016/j.pnpbp. 2012.08.009

Lees, T., Khushaba, R., and Lal, S. (2016). Electroencephalogram associations to cognitive performance in clinically active nurses. Physiol. Meas. 37, 968-980. doi: 10.1088/0967-3334/37/7/968

Li, X. Y., Yang, X. L., and Sun, Z. K. (2020). a rhythm slowing in a modified thalamocortico-thalamic model related with Alzheimer's disease. PLoS One 15:e0229950. doi: 10.1371/journal.pone.0229950

Lo, A., Chernoff, H., Zheng, T., and Lo, S. H. (2015). Why significant variables aren't automatically good predictors. Proc. Natl. Acad. Sci. U S A 112 , 13892-13897. doi: 10.1073/pnas.1518285112

Mathalon, D. H., Bennett, A., Askari, N., Max Gray, E., Rosenbloom, M. J., and Ford, J. M. (2003). Response-monitoring dysfunction in aging and Alzheimer's disease: an event-related potential study. Neurobiol. Aging 24, 675-685. doi: 10.1016/s0197-4580(02)00154-9

McKhann, G. M., Knopman, D. S., Chertkow, H., Hyman, B. T., Jack, C. R., Kawas, C. H., et al. (2011). The diagnosis of dementia due to Alzheimer's disease: recommendations from the national institute on aging-Alzheimer's association workgroups on diagnostic guidelines for Alzheimer's disease. Alzheimers Dement. 7, 263-269. doi: 10.1016/j.jalz.2011. 03.005

Meghdadi, A. H., Karić, M. S., McConnell, M., Rupp, G., Richard, C., Hamilton, J., et al. (2021). Resting state EEG biomarkers of cognitive decline associated with Alzheimer's disease and mild cognitive impairment. PLoS One 16:e244180. doi: 10.1371/journal.pone.0244180

Muliyala, K. P., and Varghese, M. (2010). The complex relationship between depression and dementia. Ann. Indian Acad. Neurol. 13, S69-S73. doi: 10.4103/0972-2327.74248

Musaeus, C. S., Engedal, K., Høgh, P., Jelic, V., Mørup, M., Naik, M., et al. (2018). EEG theta power is an early marker of cognitive decline in dementia due to Alzheimer's disease. J. Alzheimers Dis. 64, 1359-1371. doi: 10.3233/JAD180300

Niedowicz, D. M., Nelson, P. T., and Murphy, M. P. (2011). Alzheimers disease: pathological mechanisms and recent insights. Curr. Neuropharmacol. 9, 674-684. doi: 10.2174/157015911798376181

Nina, B., Florian, H., Habib, B., Ehrensperger Michael, M., Ute, G., Martin, H., et al. (2014). Slowing of EEG background activity in Parkinson's and Alzheimer's disease with early cognitive dysfunction. Front. Aging Neurosci. 6:314. doi: 10.3389/fnagi.2014.00314

Noh, G.-J., Kim, K.-M., Jeong, Y.-B., Jeong, S.-W., Yoon, H.-S., Jeong, S.M., et al. (2006). Electroencephalographic approximate entropy changes in healthy volunteers during remifentanil infusion. Anesthesiology 104, 921-932. doi: 10.1097/00000542-200605000-00006

Olichney, J. M., Yang, J.-C., Taylor, J., and Kutas, M. (2011). Cognitive eventrelated potentials: biomarkers of synaptic dysfunction across the stages of Alzheimer's disease. J. Alzheimers Dis. 26, 215-228. doi: 10.3233/JAD-20110047

Papaliagkas, V., Kimiskidis, V., Tsolaki, M., and Anogianakis, G. (2008). Usefulness of event-related potentials in the assessment of mild cognitive impairment. BMC Neurosci. 9:107. doi: 10.1186/1471-2202-9-107

Pedraza, O., Clark, J. H., O’Bryant, S. E., Smith, G. E., Ivnik, R. J., Graff-Radford, N. R., et al. (2013). Diagnostic validity of age and education corrections for the mini-mental state examination in older african americans. J. Am. Geriatr. Soc. 60, 328-331. doi: 10.1111/j.1532-5415.2011. 03766.x

Pérez-González, D., and Malmierca, M. S. (2014). Adaptation in the auditory system: an overview. Front. Integr. Neurosci. 8:19. doi: 10.3389/fnint.2014. 00019

Polich, J., and Kok, A. (1995). Cognitive and biological determinants of P300: an integrative review. Biol. Psychol. 41, 103-146. doi: 10.1016/03010511(95)05130-9

Pratt, H. (2012). "Sensory ERP components," in The Oxford Handbook of Eventrelated Potential Components, eds E. S. Kappenman and S. J. Luck (New York, NY: Oxford University Press), 1-3.

Qin, H.-Y., Zhao, X.-D., Zhu, B.-G., and Hu, C.-P. (2020). Demographic factors and cognitive function assessments associated with mild cognitive impairment progression for the elderly. Biomed Res. Int. 2020:3054373. doi: $10.1155 / 2020 / 3054373$ 
R Core Team. (2020). R: A Language and Environment for Statistical Computing. Vienna, Austria: R Foundation for Statistical Computing. Available online at: https://www.R-project.org/.

Raicher, I., Yasumasa Takahashi, D., Medeiros Kanda, P. A., Nitrini, R., and Anghinah, R. (2008). QEEG spectral peak in Alzheimer's disease: a possible tool for treatment follow-up. Dement. Neuropsychol. 2, 9-12. doi: 10.1590/S198057642009DN20100003

Rigters, S. C., Metselaar, M., Wieringa, M. H., Baatenburg de Jong, R. J., Hofman, A., and Goedegebure, A. (2016). Contributing determinants to hearing loss in elderly men and women: results from the population-based rotterdam study. Audiol. Neurootol. 21, 10-15. doi: 10.1159/000448348

Robinson, L., Tang, E., and Taylor, J. P. (2015). Dementia: timely diagnosis and early intervention. BMJ 350:h3029. doi: 10.1136/bmj.h3029

Rodriguez, G., Arnaldi, D., and Picco, A. (2011). Brain functional network in Alzheimer's disease: diagnostic markers for diagnosis and monitoring. Int. J. Alzheimers Dis. 2011:481903. doi: 10.4061/2011/481903

Roh, S.-C., Kim, J. S., Kim, S., Kim, Y., and Lee, S. H. (2020). Frontal $\alpha$ asymmetry moderated by suicidal ideation in patients with major depressive disorder: a comparison with healthy individuals. Clin. Psychopharmacol. Neurosci. 18, 58-66. doi: 10.9758/cpn.2020.18.1.58

Rossini, P. M., Buscema, M., Capriotti, M., Grossi, E., Rodriguez, G., Del Percio, C., et al. (2008). Is it possible to automatically distinguish resting EEG data of normal elderly vs. mild cognitive impairment subjects with high degree of accuracy? Clin. Neurophysiol. 119, 1534-1545. doi: 10.1016/j.clinph.2008.03. 026

Rossini, P. M., Di Iorio, R., Vecchio, F., Anfossi, M., Babiloni, C., Bozzali, M., et al. (2020). Early diagnosis of Alzheimer's disease: the role of biomarkers including advanced EEG signal analysis. report from the IFCN-sponsored panel of experts. Clin. Neurophysiol. 131, 1287-1310. doi: 10.1016/j.clinph. 2020.03.003

Salisbury, D. F., Rutherford, B., Shenton, M. E., and McCarley, R.-W. (2001). Button-pressing affects P300 amplitude and scalp topography. Clin. Neurophysiol. 112, 1676-1684. doi: 10.1016/s1388-2457(01)00607-1

Scazufca, M., Almeida, O. P., Vallada, H. P., Tasse, W. A., and Menezes, P. R. (2009). Limitations of the mini-mental state examination for screening dementia in a community with low socioeconomic status: results from the sao paulo ageingand health study. Eur. Arch. Psychiatry Clin. Neurosci. 259, 8-15. doi: 10.1007/s00406-008-0827-6

Schmidt, M. T., Kanda, P. A. M., Basile, L. F. H., da Silva Lopes, H. F., Baratho, R., Demario, J. L. C., et al. (2013). Index of $\alpha /$ theta ratio of the electroencephalogram: a new marker for Alzheimer's disease. Front. Aging Neurosci. 5:60. doi: 10.3389/fnagi.2013.00060

Smailovic, U., and Jelic, V. (2019). Neurophysiological markers of Alzheimer's disease: quantitative EEG approach. Neurol. Ther. 8, 37-55. doi: 10.1007/s40120-019-00169-0

Sperling, R. A., Aisen, P. S., Beckett, L. A., Bennet, D. A., Craft, S., Fagan, A. M., et al. (2011). Toward defining the preclinical stages of Alzheimer's disease: recommendations from the national institute on aging. Alzheimers Dement. 7 , 280-292. doi: 10.1016/j.jalz.2011.03.003

Tae, H. K., Jin, H. J., Joon, H. P., Jeong, L. K., Seung, H. R., Seok, W. M., et al. (2010). Korean version of mini mental status examination for dementia screening and its short form. Psychiatry Investig. 7, 102-108. doi: 10.4306/pi. 2010.7.2.102

Tanaka, F., Kachi, T., Yamada, T., and Sobue, G. (1998). Auditory and visual event-related potentials and flash visual evoked potentials in Alzheimer's disease: correlations with Mini-Mental State Examination and Raven's Coloured Progressive Matrices. J. Neurol. Sci. 156, 83-88. doi: 10.1016/s0022510x(98)00004-5

Thibodeau, R., Jorgensen, R. S., and Kim, S. (2006). Depression, anxiety, and resting frontal EEG asymmetry: a meta-analytic review. J. Abnorm. Psychol. 115, 715-729. doi: 10.1037/0021-843X.115.4.715
Tisher, A., and Salardini, A. (2019). A comprehensive update on treatment of dementia. Semin. Neurol. 39, 167-178. doi: 10.1055/s-0039-1683408

Tsoi, K. K. F., Chan, J. Y. C., Hirai, H. W., Wong, S. Y. S., and Kwok, T. C. Y. (2015). Cognitive tests to detect dementia a systematic review and metaanalysis. JAMA Intern. Med. 175, 1450-1458. doi: 10.1001/jamainternmed. 2015.2152

Uhlhaas, P. J., and Singer, W. (2006). Neural synchrony in brain disorders: relevance for cognitive dysfunctions and pathophysiology. Neuron 52, 155-168. doi: 10.1016/j.neuron.2006.09.020

van der Vinne, N., Vollebregt, M. A., van Putten, M. J. A. M., and Arns, M. (2017). Frontal $\alpha$ asymmetry as a diagnostic marker in depression: fact or fiction? A meta-analysis. Neuroimage Clin. 16, 79-87. doi: 10.1016/j.nicl.2017. 07.006

van Deursen, J. A., Vuurman, E. F., Smits, L. L., Verhey, F. R., and Riedel, W. J. (2009). Response speed, contingent negative variation and P300 in Alzheimer's disease and MCI. Brain Cogn. 69, 592-599. doi: 10.1016/j.bandc.2008.12.007

van Vliet, M., Manyakov, N. V., Storms, G., Fias, W., Wiersema, J. R., and Van Hulle, M. M. (2014). Response-related potentials during semantic priming: the effect of a speeded button response task on ERPs. PLoS One 9:e87650. doi: 10.1371/journal.pone.0087650

Vecchio, F., and Määttä, S. (2011). The use of auditory event-related potentials in Alzheimer's disease diagnosis. Int. J. Alzheimers Dis. 2011:653173. doi: $10.4061 / 2011 / 653173$

Vecchio, F., Lizio, R., Frisoni, G. B., Ferri, R., Rodriguez, G., and Babiloni, C. (2011). Electroencephalographic rhythms in Alzheimer's disease. Int. J. Alzheimers Dis. 2011:927573. doi: 10.4061/2011/927573

Williams, P. A., Jones, G. H., Briscoe, M., Thomas, R., and Cronin, P. (1991). $\mathrm{P} 300$ and reaction-time measures in senile dementia of the Alzheimer type. $\mathrm{Br}$. J. Psychiatry 159, 410-414. doi: 10.1192/bjp.159.3.410

Wong, T. T., and Yeh, P. Y. (2020). Reliable accuracy estimates from K-fold cross validation. IEEE Trans. Knowl. Data Eng. 32, 1586-1594. doi: 10.1109/tkde. 2019.2912815

Woodman, G. F. (2010). A brief introduction to the use of event-related potentials in studies of perception and attention. Atten. Percept. Psychophys. 72, 2031-2046. doi: 10.3758/APP.72.8.2031

World Health Organization. (1992). International Statistical Classification of Diseases and Related Health. Problems. 10th Edn. World Health Organization.

World Health Organization. (2020). Dementia. World Health Organization. Available online at: https://www.who.int/news-room/factsheets/detail/dementia.

Wright, M. N., Wager, S., and Probst, P. (2020). Ranger: A Fast Implementation of Random Forests. Available online at: https://CRAN.Rproject.org/package $=$ ranger.

Yener, G. G., and Başar, E. (2010). Sensory evoked and event related oscillations in Alzheimer's disease: a short review. Cogn. Neurodyn. 4, 263-274. doi: 10.1007/s11571-010-9138-5

Zvěřová, M. (2018). Alzheimer's disease and blood-based biomarkers-potential contexts of use. Neuropsychiatr. Dis. Treat. 14, 1877-1882. doi: 10.1016/j.clnu. 2021.02.024

Conflict of Interest: The authors declare that the research was conducted in the absence of any commercial or financial relationships that could be construed as a potential conflict of interest.

Copyright (c) 2021 Doan, Ku, Choi, Oh, Kim, Cha and Kim. This is an open-access article distributed under the terms of the Creative Commons Attribution License (CC BY). The use, distribution or reproduction in other forums is permitted, provided the original author(s) and the copyright owner(s) are credited and that the original publication in this journal is cited, in accordance with accepted academic practice. No use, distribution or reproduction is permitted which does not comply with these terms. 


\section{APPENDIX}

TABLE A1 | Estimated odds ratios and 95\% confidence intervals derived from three logistic regression models.

Unadjusted

Wald Z
(p-value)

Adjusted

Wald

$-2.26(2.39-102)$

Alpha (avg.) $\left[\mu \mathrm{V}^{2}\right]$

Alpha/Theta

Beta (avg.) $\left[\mu \mathrm{V}^{2}\right]$

Frontal asymmetry

Peak frequency $[\mathrm{Hz}]$

MEF $[\mathrm{Hz}]$

Peak power $\left[\mu \mathrm{V}^{2}\right]$

Theta (avg.) $\left[\mu \mathrm{V}^{2}\right]$

Sensory ERP

Amplitude (deviation) $[\mu \mathrm{V}]$

Amplitude (edge-center ratio)

Amplitude $[\mu \mathrm{V}]$

Response time (deviation) [ms]

Attention ERP

Response time [ms]

$0.615(0.40,0.93)$
$0.289(0.16,0.49)$
$1.355(0.91,2.05)$
$0.546(0.34,0.83)$
$0.255(0.14,0.42)$
$0.285(0.17,0.45)$
$0.969(0.61,1.42)$
$1.699(1.14,2.60)$

$1.459(0.99,2.19)$
$1.094(0.74,1.63)$
$0.931(0.62,1.38)$
$1.424(0.97,2.11)$
$1.967(1.32,3.01)$

$-4.39(1.14 \mathrm{E}-05)$

$1.49(1.37 \mathrm{E}-01)$

$-2.68(7.47 \mathrm{E}-03)$

$-5.01(5.58 \mathrm{E}-07)$

$-4.97(6.64 \mathrm{E}-07)$

-0.15 (8.79E-01)

2.54 (1.09E-02)

$(95 \% \mathrm{Cl})$

$1.88(5.95 \mathrm{E}-02)$

0.45 (6.56E-01)

-0.35 (7.24E-01)

1.79 (7.32E-02)

$1.967(1.32,3.01)$

$3.23(1.25 \mathrm{E}-03)$

\# of correct

Amp (resp) - Amp (bg) $[\mu \mathrm{V}]$

Resp. Time [s]

$0.521(0.34,0.77)$

$0.349(0.13,0.75)$

$2.130(1.42,3.32)$

$-3.18(1.46 \mathrm{E}-03)$

$-2.33(1.96 \mathrm{E}-02)$

$3.53(4.21 \mathrm{E}-04)$

$2.364(1.59,3.66)$

4.09 (4.31E-05)
$0.693(0.42,1.12)$

$0.474(0.24,0.87)$

$1.186(0.74,1.92)$

$0.516(0.30,0.82)$

$0.387(0.20,0.69)$

$0.387(0.21,0.68)$

$0.868(0.48,1.45)$

$1.143(0.70,1.89)$

$1.282(0.80,2.12)$

$0.903(0.57,1.43)$

$0.875(0.54,1.40)$

$1.538(0.95,2.51)$

$2.109(1.31,3.56)$

$0.711(0.45,1.10)$

$0.626(0.23,1.20)$

$1.593(1.01,2.59)$
Adjusted (including MMSE)

OR
$(95 \% \mathrm{Cl})$

Wald Z

(p-value)

$0.747(0.43,1.26)$

$-1.47(1.41 \mathrm{E}-01)$

-2.33 (1.99E-02)

0.71 (4.76E-01)

$-2.58(9.75 E-03)$

-3.07 (2.11E-03)

$-3.19(1.40 \mathrm{E}-03)$

-0.51 (6.13E-01)

0.53 (5.94E-01)

1.01 (3.13E-01)

-0.44 (6.61E-01)

-0.56 (5.78E-01)

1.75 (7.96E-02)

2.96 (3.09E-03)

-1.54 (1.24E-01)

$-1.13(2.59 \mathrm{E}-01)$

1.96 (5.04E-02)

1.75 (8.02E-02)

$0.747(0.43,1.26)$

$0.630(0.32,1.21)$

$1.204(0.72,2.04)$

$0.535(0.30,0.87)$

$0.460(0.24,0.83)$

$0.555(0.28,1.06)$

$0.842(0.46,1.43)$

$0.952(0.55,1.65)$

$1.213(0.71,2.07)$

$0.884(0.53,1.47)$

$0.768(0.44,1.30)$

$1.292(0.76,2.17)$

$1.892(1.15,3.26)$

$1.106(0.65,1.97)$

$0.844(0.32,1.57)$

$1.408(0.86,2.34)$

$0.950(0.52,1.69)$

$-1.08(2.80 \mathrm{E}-01)$

-1.36 (1.75E-01)

$0.71(4.78 \mathrm{E}-01)$

-2.29 (2.21E-02)

-2.50 (1.26E-02)

-1.76 (7.79E-02)

-0.60 (5.52E-01)

-0.18 (8.60E-01)

0.72 (4.72E-01)

-0.48 (6.33E-01)

-0.97 (3.32E-01)

0.96 (3.35E-01)

2.42 (1.55E-02)

0.36 (7.19E-01)

-0.43 (6.70E-01)

$1.36(1.74 \mathrm{E}-01)$

-0.17 (8.61E-01)

The table shows the exact values for Figure 4. Due to the small sample size in this study, the covariates related to the disease status (e.g., hypertension, diabetes, and so on) were not included in the multiple logistic regression model. OR (95\% Cl), Odds ratios with 95\% confident interval; Wald Z (p-value), P-value obtains from Wald test. 